\begin{tabular}{|c|l|}
\hline Title & Slowly decaying solutions for semilinear wave equations in odd space dimensions \\
\hline Author(s) & Kubo, H. \\
\hline Citation & Hokkaido University Preprint Series in Mathematics, 245, 1-30 \\
\hline Issue Date & 19945-1 \\
\hline DOI & 10.14943/83392 \\
\hline Doc URL & http://hdl.handle.net/2115/68996 \\
\hline Type & bulletin (article) \\
\hline File Information & pre245.pdf \\
\hline
\end{tabular}

Instructions for use 
SLOWLY DECAYING SOLUTIONS

FOR SEMILINEAR WAVE

EQUATIONS IN ODD

SPACE DIMENSIONS

Hideo Kubo

Series $\sharp 245$. May 1994 


\section{HOKKAIDO UNIVERSITY \\ PREPRINT SERIES IN MATHEMATICS}

\#219: Y. Giga, K. Yama-uchi, On instability of evolving hypersurfaces, 14 pages. 1993.

\#220: W. Bruns, T. Hibi, Cohen-Macaulay partially ordered sets with pure resolutions, 11 pages. 1993.

\#221: S. Jimbo, Y. Morita, Ginzburg Landau equation and stable solutions in a rotational domain, 32 pages. 1993.

\#222: T. Miyake, Y. Maeda, On a property of Fourier coefficients of cusp forms of half-integral weight, 12 pages. 1993.

$\sharp 223$ : I. Nakai, Notes on versal deformation of first order PDE and web structure, 34 pages. 1993.

$\sharp 224$ : I. Tsuda, Can stochastic renewal of maps be a model for cerebral cortex?, 30 pages. 1993.

$\sharp 225$ : H. Kubo, K. Kubota, Asymptotic behaviors of radial solutions to semilinear wave equations in odd space dimensions, 47 pages. 1994.

\# 226: T. Nakazi, K. Takahashi, Two dimensional representations of uniform algebras, 7 pages. 1994.

$\sharp 227$ : N. Hayashi, T. Ozawa, Global, small radially symmetric solu tions to nonlinear Schrödinger equations and a gauge transformation, 16 pages. 1994.

$\sharp 228$ : S. Izumiya, Characteristic vector fields for first order partial differential equations, 9 pages. 1994.

$\sharp 229$ : K. Tsutaya, Lower bounds for the life span of solutions of semilinear wave equations with data of non compact support, 14 pages. 1994.

\#230: H. Okuda, I. Tsuda, A coupled chaotic system with different time scales: Toward the implication of observation with dynamical systems, 31 pages. 1994 .

$\sharp 231$ : A. Hoshiga, The asymptotic behaviour of radial solutions near the blow-up point to quasi-linear wave equations in two space dimensions, 8 pages. 1994.

\#232: Y. Giga, N. Mizoguchi, Existence of periodically evolving convex curves moved by anisotropic curvature, 12 pages. 1994.

$\sharp 233$ : C. Dohmen, Y. Giga, N. Mizoguchi, Existence of selfsimilar shrinking curves for anisotropic curvature flow equations, 13 pages. 1994.

\#234: T. Nakazi, M. Yamada, Invertible Toeplitz operators and uniform algebras, 14 pages. 1994.

\#235: C. Dohmen, A Pohozaev-type inequality for a quasilinear Haraux-Weissler equation, 6 pages. 1994.

\#236: T. Mikami, Large deviations for the first exit time on small random perturbations of dynamical systems, 32 pages. 1994.

$\sharp 237$ T. Ozawa, Characterization of Trudinger's inequality, 7 pages. 1994.

\#238 T. Hibi, Buchsbaum complexes with linear resolutions, 12 pages. 1994.

$\sharp 239$ Y. Giga, N. Mizoguchi, On time periodic solutions of the Dirichlet problem for degenerate parabolic equations of nondivergence type, 23 pages. 1994.

$\sharp 240$ C. Dohmen, Existence of Fast Decaying Solutions to a Haraux-Weissler Equation With a Prescribed Number of Zeroes, 12 pages. 1994.

$\sharp 241$ K. Sugano, Note on H-separable Frobenius extensions, 8 pages. 1994.

\# 242 J. Zhai, Some Estimates For The Blowing up Solutions of Semilinear Heat Equations, 11 pages. 1994.

$\sharp 243$ N. Hayashi, K. Kato and T. Ozawa, Dilation Method and Smoothing Effect of the Schrödinger Evolution Group, 10 pages. 1994.

\#244 D. Lehmann, T. Suwa, Residues of holomorphic vector fields relative to singular invariant subvarieties, 26 pages. 1994. 


\title{
SLOWLY DECAYING SOLUTIONS FOR SEMILINEAR WAVE EQUATIONS IN ODD SPACE DIMENSIONS
}

\author{
Hideo Kubo \\ Department of Mathematics Hokkaido University, Sapporo 060, Japan
}

1. Introduction and Statements of Results. In this paper we consider the initial value problem to a nonlinear wave equation:

$$
u_{t t}-\Delta_{x} u=|u|^{p} \text { in } \mathbb{R}_{x}^{n} \times[0, \infty)
$$

where $u$ is a real valued function and $p>1$. It is a basic problem whether or not the initial value problem to (1.1) admits a unique global solution. If the initial data is large in a certain sense, there is no global in time solution to the problem for any $p>1$. (See for instance R.T. Glassey [7]).

For small initial data, F. John [10] established a remarkable result when space dimensions $n=3$, namely, he showed there is a 'critical' value $p_{0}(3)$; where $p_{0}(n)$ is the positive root of the quadratic equation:

$$
(n-1) p^{2}-(n+1) p-2=0
$$

Here critical means that a unique global solution exists if $p>p_{0}(n)$ and any nontrivial classical solution blows up in finite time if $1<p<p_{0}(n)$ under suitable assumptions on the initial data. Glassey [8], [9] also proved that this assertion is still valid when $n=2$. For the critical case $p=p_{0}(n), n=2,3, \mathrm{~J}$. Schaeffer [18] obtained the blow-up result. By virtue of these works, we find a relation between a global behavior of the classical solutions and the powcr of the nonlinearity, if the initial data are 'nice'. As for subcritical case, i.e., $1<p<p_{0}(n)$, T.C. Sideris [19] extended the nonexistence result in general space dimensions. When $n=4$, Y. Zhou [25] constructed a unique global solution in a generalized Sobolev spaces provided $p>p_{0}(4)$ and the initial data are rapidly decaying. Moreover there are many works which contribute to the initial value problem. (See for example [12], [4], [16], [3] and [13]). However the case where $p \geq p_{0}(n), n \geq 5$ and $p=p_{0}(4)$ is still open. 
On the other hand, the decay rate of the initial data as well as the power $p$ plays an important role to determine a global behavior of the classical solution to the initial value problem for (1.1) with prescribed the following initial condition:

$$
u(x, 0)=f(x), \quad u_{t}(x, 0)=g(x) \quad \text { for } \quad x \in \mathbb{R}^{n}
$$

where $f \in C^{[n / 2]+2}\left(\mathbb{R}^{n}\right), g \in C^{[n / 2]+1}\left(\mathbb{R}^{n}\right)$. We suppose either

$$
\sum_{|\alpha| \leq[n / 2]+2}\left|\partial_{x}^{\alpha} f(x)\right|+\sum_{|\alpha| \leq[n / 2]+1}\left|\partial_{x}^{\alpha} g(x)\right| \leq \varepsilon(1+|x|)^{-(\kappa+1)}
$$

or

$$
f \equiv 0, \quad g(x) \geq \varepsilon(1+|x|)^{-(\kappa+1)}
$$

where $\kappa$ and $\varepsilon$ are positive parameters. In three space dimensions, F. Asakura [3] found a critical value of $\kappa$, namely, if $\kappa>\kappa_{0}=2 /(p-1)$ and $p>p_{0}(3)$, a unique global solution exists by assuming (1.4) with $\varepsilon$ sufficiently small and if $\kappa<\kappa_{0}$ and (1.5) holds, any classical solution blows up in finite time. This remarkable phenomenon was also studied for two space dimensional case. The existence part is proved by K. Kubota [15], K. Tsutaya [23] independently. Moreover when $\kappa=\kappa_{0}$ and $n=2,3$, the problem admits a unique global solution, provided (1.4) holds and $\varepsilon$ is sufficiently small. (See for instance [15], Tsutaya [22] and [24]). On the other hand, the blow-up part is established by R. Agemi and H. Takamura [2], [23], independently. The critical value is the same number as in [3]. When $n \geq 4$, Takamura [20], [21] proved that if $\kappa<\kappa_{0}$ and (1.5) hold, the blow-up occurs in finite time for radially symmetric case. Moreover the following upper bound of the lifespan is obtained by [2], [22], [24], [20] and [21]:

$$
T_{\varepsilon} \leq C \varepsilon^{-(p-1) /(2-(p-1) \kappa)}
$$

where lifespan means the maximal time interval where classical solutions exist uniquely and $C=C(n, p, \kappa)>0$.

Then the following question naturally arises : Whether or not the value $\kappa_{0}$ is also 'critical' for the higher space dimensional case. The aim of this paper is to answer this question affirmatively, provided the initial data are radially symmetric, the space dimensions is odd and $p>\tilde{p_{0}}(n) \equiv \max \left(p_{0}(n),(n-3) / 2\right)$. The last assumption implies that there is a gap between the critical number $p_{0}(n)$ and $\tilde{p_{0}}(n)$ except five space dimensional case. 
We now rewrite the equation (1.1) and the initial condition (1.3) as follows:

$$
\begin{aligned}
& u_{t t}-u_{r r}-\frac{n-1}{r} u_{r}=|u|^{p} \quad \text { in } \mathbb{R} \times[0, \infty), \\
& u(r, 0)=f(r), \quad u_{t}(r, 0)=g(r) \text { for } r \in \mathbb{R},
\end{aligned}
$$

where $f$ and $g$ are even functions such that $f \in C^{m+1}(\mathbb{R}), g \in C^{m}(\mathbb{R})$ and $n=2 m+3$ with $m$ a positive integer. Moreover we assume that

$$
|f(r)|\langle r\rangle^{\kappa}+\sum_{j=0}^{m}\left\{\left|f^{(j+1)}(r)\right|+\left|g^{(j)}(r)\right|\right\}\langle r\rangle^{1+\kappa+j} \leq \varepsilon
$$

where $\kappa, \varepsilon$ are positive parameters. Besides, we have set $\langle r\rangle=\sqrt{1+r^{2}}, g^{(j)}(r)=\left(\frac{d}{d r}\right)^{j} g(r)$. As to the power $p$, we suppose

$$
p>p_{0}(n) \text { and } p>m=\frac{n-3}{2} .
$$

We slightly modify the differentiabilty of solutions in the definition of $T_{\varepsilon}$ as follows: $u \in C(\mathbb{R} \times[0, T))$ and $r^{2} u \in C^{2}(\mathbb{R} \times[0, T))$, particularly, $u \in C^{2}((\mathbb{R} \backslash\{0\}) \times[0, T))$.

Then our main result is

Theorem 1.1. Suppose that (1.8), (1.9) hold. Then there is a positive number $\varepsilon_{0}=$ $\varepsilon_{0}(n, p, \kappa)$ such that for $0<\varepsilon \leq \varepsilon_{0}$

$$
\begin{aligned}
& T_{\varepsilon}=\infty \quad \text { if } \quad \kappa \geq \kappa_{0}=2 /(p-1), \\
& T_{\varepsilon} \geq C \varepsilon^{-(p-1) /(2-(p-1) \kappa)} \quad \text { if } 0<\kappa<\kappa_{0},
\end{aligned}
$$

where $C=C(n, p, \kappa)>0$.

One can easily generalize Theorem 1.1 as follows. We consider the following equation:

$$
u_{t t}-u_{r r}-\frac{n-1}{r} u_{r}=G(u) \text { in } \mathbb{R} \times[0, \infty) .
$$

We impose the following hypothesis $(\mathrm{H})$ on $G$ :

$$
\begin{aligned}
& G(\lambda) \in C^{m}(\mathbb{R}) \text { and there are constants } p>p_{0}(n), A>0 \text { such that } \\
& \left|G^{(l)}(\lambda)\right| \leq A|\lambda|^{p-l} \text { if } l<p, l \leq m \text { and }|\lambda| \leq 1 .
\end{aligned}
$$

Moreover there are constants $B>0$ and $0<\delta \leq 1$ such that for $|\lambda|,\left|\lambda^{\prime}\right| \leq 1$

$$
\left|G^{(m)}(\lambda)-G^{(m)}\left(\lambda^{\prime}\right)\right| \leq\left\{\begin{array}{lc}
B\left|\lambda-\lambda^{\prime}\right|\left(|\lambda|^{p-(m+1)}+\left|\lambda^{\prime}\right|^{p-(m+1)}\right) \quad \text { if } p>m+1, \\
B\left|\lambda-\lambda^{\prime}\right|^{p-m} & \text { if } m<p \leq m+1, \\
B\left|\lambda-\lambda^{\prime}\right|^{\delta} & \text { if } p \leq m .
\end{array}\right.
$$

A typical examples is $G(u)=u^{2}$ if $m \geq 2$ and $G(u)=|u|^{p}$ if $m=1$ and $p>p_{0}(5)$. 
Theorem 1.2. Suppose that (1.8), (H) hold. Then there is a positive number $\varepsilon_{1}=\varepsilon_{1}(n, G, \kappa)$ such that (1.10) with $C$ replaced by $C^{\prime}=C^{\prime}(n, G, \kappa)>0$ holds for $0<\varepsilon \leq \varepsilon_{1}$.

This paper is organized as follows. First we shall study a solution to a linear wave equation in Section 2. Section 3 is devoted to obtain basic a priori estimates. In the final section we shall construct a global solution by employing the iteration method and obtain the lower bound of the lifespan.

Throughout of this paper, we shall denote various constants, depending only on $n, G$ and $\kappa$ by $C, C_{0}, C_{1}$ and so on.

2. Linear Wave Equations. The aim of this section is to obtain preliminary decay estimates for a solution to a linear wave equation:

$$
u_{t t}-u_{r r}-\frac{n-1}{r} u_{r}=0 \text { in } \mathbb{R} \times[0, \infty)
$$

To begin with, we define

$$
v(r, t)=\int_{-1}^{1} H(t+r \sigma) \psi(\sigma) d \sigma, \quad \psi(\sigma)=\left(1-\sigma^{2}\right)^{m}, m=(n-3) / 2 .
$$

If $H(\rho)$ belongs to $C^{2}(\mathbb{R})$, then $v(r, t)$ belongs to $C^{2}(\mathbb{R} \times[0, \infty))$ and satisfies $(2.1)$. (See e.g. [13], Lemma 2.1). However we modify it as follows:

Lemma 2.1. Suppose that $H(\rho) \in C(\mathbb{R})$ and $m \in \mathbb{N}$. Then $v(r, t)$ is even in $r$ and $\partial_{r}^{j}\left(r^{j} v\right)(r, t)$ belongs to $C(\mathbb{R} \times[0, \infty)), 0 \leq j \leq m+1$. Moreover we have

$$
\begin{aligned}
\partial_{r}^{j}\left(r^{j} v\right)(r, t)= & \int_{-1}^{1} H(t+r \sigma)(-\sigma)^{j} \psi^{(j)}(\sigma) d \sigma, \quad 0 \leq j \leq m \\
\partial_{r}^{m+1}\left(r^{m+1} v\right)(r, t)= & \int_{-1}^{1} H(t+r \sigma)(-\sigma)^{m+1} \psi^{(m+1)}(\sigma) d \sigma \\
& +(-1)^{m} H(t+r) \psi^{(m)}(1)+H(t-r) \psi^{(m)}(-1) .
\end{aligned}
$$

Furthermore $r^{2} v(r, t)$ belongs to $C^{2}(\mathbb{R} \times[0, \infty))$ and satisfies

$$
r^{2} v_{t t}-r^{2} v_{r r}-(n-1) r v_{r}=0 \text { in } \mathbb{R} \times[0, \infty)
$$

Proof: It is easy to see that $v$ is even in $r$. Next we consider (2.3). If we change the variable by $\rho=t+r \sigma$, the result is

$$
r v(r, t)=\int_{t-r}^{t+r} H(\rho) \psi(\sigma) d \rho, \quad r \neq 0
$$


Since $\psi( \pm 1)=0$, we have

$$
\partial_{r}(r v)(r, t)=\int_{t-r}^{t+r} H(\rho) \psi^{(1)}(\sigma) \sigma_{r} d \rho=\int_{-1}^{1} H(t+r \sigma)(-\sigma) \psi^{(1)}(\sigma) d \sigma
$$

because $\sigma_{r}=-\sigma / r$. Therefore (2.3) holds for $j=1$.

We shall show (2.3) by employing the induction, namely, we assume the following identity holds for some $j$ with $1 \leq j \leq m$ :

$$
\partial_{r}^{j}\left(r^{j} v\right)(r, t)=\frac{1}{r} \int_{t-r}^{t+r} H(\rho)(-\sigma)^{j} \psi^{(j)}(\sigma) d \rho, \quad r \neq 0 .
$$

By Leibnitz' rule, we have

$$
\begin{aligned}
\partial_{r}^{j+1}\left(r \cdot r^{j} v\right)(r, t) & =r \partial_{r}^{j+1}\left(r^{j} v\right)(r, t)+(j+1) \partial_{r}^{j}\left(r^{j} v\right)(r, t) \\
& =\partial_{r}\left(r \partial_{r}^{j}\left(r^{j} v\right)\right)(r, t)+j \partial_{r}^{j}\left(r^{j} v\right)(r, t)
\end{aligned}
$$

Using the assumption of the induction, we have

$$
\begin{aligned}
\partial_{r}\left(r \partial_{r}^{j}\left(r^{j} v\right)\right)(r, t)= & \partial_{r} \int_{t-r}^{t+r} H(\rho)(-\sigma)^{j} \psi^{(j)}(\sigma) d \rho \\
= & \int_{t-r}^{t+r} H(\rho)(-\sigma)^{j} \psi^{(j+1)}(\sigma) \sigma_{r} d \rho \\
& +j \int_{t-r}^{t+r} H(\rho)(-\sigma)^{j-1} \psi^{(j)}(\sigma)(-\sigma)_{r} d \rho \\
& +(-1)^{j} H(t+r) \psi^{(j)}(1)+H(t-r) \psi^{(j)}(-1)
\end{aligned}
$$

Since $\psi^{(j)}( \pm 1)=0,1 \leq j \leq m-1$, combining this with (2.7) together with the assumption of the induction, we obtain (2.3).

Finally we shall show (2.4). We note that

$$
\left(1-\sigma^{2}\right) \psi^{(2)}(\sigma)+(n-5) \sigma \psi^{(1)}(\sigma)+(n-3) \psi(\sigma)=0
$$

which is easily checked by a simple calculation. In a similar way to get (2.6), we have

$$
r \partial_{t}(r v)(r, t)=-\int_{t-r}^{t+r} H(\rho) \psi^{(1)}(\sigma) d \rho, \quad r \neq 0
$$

which yields

$$
\begin{aligned}
\partial_{t}\left(r \partial_{t}(r v)(r, t)\right)= & -\int_{t-r}^{t+r} H(\rho) \psi^{(2)}(\sigma)\left(-\frac{1}{r}\right) d \rho \\
& -H(t+r) \psi^{(1)}(1)+H(t-r) \psi^{(1)}(-1) \\
= & \int_{-1}^{1} H(t+r \sigma) \psi^{(2)}(\sigma) d \sigma \\
& -H(t+r) \psi^{(1)}(1)+H(t-r) \psi^{(1)}(-1)
\end{aligned}
$$


Since

$$
\begin{aligned}
& r^{2} \partial_{t}^{2} v(r, t)-r^{2} \partial_{r}^{2} v(r, t)-(n-1) r \partial_{r} v(r, t) \\
= & r^{2} \partial_{t}^{2} v(r, t)-\left\{\partial_{r}^{2}\left(r^{2} v\right)(r, t)+(n-5) \partial_{r}(r v)(r, t)-(n-3) v(r, t)\right\}
\end{aligned}
$$

by virtue of (2.10), (2.3) and (2.9), we obtain (2.4).

We now define a function $u^{0}$ by

$$
u^{0}(r, t)=\int_{-1}^{1} H_{g}(t+r \sigma) \psi(\sigma) d \sigma+\partial_{t} \int_{-1}^{1} H_{f}(t+r \sigma) \psi(\sigma) d \sigma
$$

where we have set $\psi(\sigma)=\left(1-\sigma^{2}\right)^{m}$ and

$$
H_{g}(\rho)=\frac{1}{2 m !}\left(\frac{1}{2 \rho} \frac{d}{d \rho}\right)^{m}\left(\rho^{2 m+1} g(\rho)\right), \quad m=(n-3) / 2
$$

Then $u^{0}$ satisfies the initial condition (1.7). (See e.g. Courant and Hilbert [6], p.699-p.703 or [13], Lemma 2.1).

Here we introduce a Banach space $X$ in which we will construct a solution of the initial value problem (1.11), (1.7):

$$
X=\left\{u(r, t) \in C(\mathbb{R} \times[0, \infty)) ; \partial_{r}^{j}\left(r^{j} u\right)(r, t) \in C(\mathbb{R} \times[0, \infty)) \text { for } 1 \leq j \leq m,\|u\|<\infty\right\}
$$

where the norm $\|u\|$ is defined according to the size of $\kappa$ given in (1.8):

(i) $l<\kappa<l+1, \quad 0 \leq l \leq m$ :

$$
\|u\|=\sum_{j=0}^{m} \sup _{(r, t)}\left|\partial_{r}^{j}\left(r^{j} u\right)(r, t)\right|\langle t+|r|\rangle^{\mu}\langle r\rangle^{\min (l, m-j)} \quad \text { with } \quad \mu=\kappa-l
$$

(ii) $\quad \kappa=l+1, \quad 0 \leq l \leq m$ :

$$
\|u\|=\sum_{j=0}^{m} \sup _{(r, t)}\left|\partial_{r}^{j}\left(r^{j} u\right)(r, t)\right|\langle t+|r|\rangle\langle r\rangle^{\min (l, m-j)} \Psi_{j-(m-l)}^{-1}(r, t)
$$

(iii) $\kappa>m+1:$

$$
\|u\|=\sum_{j=0}^{m} \sup _{(r, t)}\left|\partial_{r}^{j}\left(r^{j} u\right)(r, t)\right|\langle t+|r|\rangle\langle r\rangle^{m-j}\langle t-|r|\rangle^{\nu} \quad \text { with } \quad \nu=\kappa-(m+1),
$$

where we have set $\Psi_{\alpha}(r, t)=\left(1+\log \frac{\langle t+|r|\rangle}{\langle t-|r|\rangle}\right)$ for $\alpha \geq 0$ and $\Psi_{\alpha}(r, t)=1$ for $\alpha<0$. Applying Lemma 2.1 as $H(\rho)=H_{g}(\rho)+H_{f}^{(1)}(\rho)$ to $u^{0}$, we obtain the following proposition. 
Proposition 2.2. Suppose (1.8) holds. Then $u^{0} \in X$ and $r^{2} u^{0} \in C^{2}(\mathbb{R} \times[0, \infty))$. Moreover $u^{0}$ satisfies (2.4), (1.7) and is even in r. Furthermore we have

$$
\|u\| \leq C_{0} \varepsilon
$$

where $C_{0}$ is a numerical constant depending only on $m$ and $\kappa$.

Proof: The assertions of the proposition except (2.12) are direct consequences of the preceding lemma, because $H_{g} \in C(\mathbb{R})$ and $H_{f} \in C^{1}(\mathbb{R})$.

In the rest of this section, we shall show (2.12). Since $u^{0}$ is even in $r$, we may assume $r>0$. First we consider the case $t \geq 2 r>0$ or $0<r \leq 1$ by making use of the identity (2.3a). Using the assumption (1.8), we have

$$
\left|H_{g}(\rho)+H_{f}^{(1)}(\rho)\right| \leq C \varepsilon\langle\rho\rangle^{-\kappa}
$$

because

$$
H_{g}(\rho)+H_{f}^{(1)}(\rho)=\sum_{j=0}^{m} C_{j} \rho^{1+j} g^{(j)}(\rho)+\sum_{j=0}^{m+1} C_{j}^{\prime} \rho^{j} f^{(j)}(\rho) .
$$

Then it follows from (2.11), (2.13) that for $r \neq 0$

$$
\left|\partial_{r}^{j}\left(r^{j} u^{0}\right)(r, t)\right| \leq \frac{C \varepsilon}{r} \int_{t-r}^{t+r}\langle\rho\rangle^{-\kappa} d \rho \leq \frac{2 C \varepsilon}{r} \int_{(t-r)_{+}}^{t+r}\langle\rho\rangle^{-\kappa} d \rho,
$$

where we have set $\alpha_{+}=\max (\alpha, 0)$. Since $\langle\rho\rangle$ is equivalent to $\langle t+r\rangle$ for $(t-r)_{+} \leq \rho \leq t+r$ if either $t \geq 2 r>0$ or $0<r \leq 1$, we get

$$
\left|\partial_{r}^{j}\left(r^{j} u^{0}\right)(r, t)\right| \leq C \varepsilon\langle t+r\rangle^{-\kappa}, \quad 0 \leq j \leq m
$$

Next we consider the case where $0<t \leq 2 r$ and $r \geq 1$. Changing the variable by $\rho=t+r \sigma$ and integrating by parts $m$ times in (2.11), we obtain

$$
u^{0}(r, t)=\int_{t-r}^{t+r} g(\rho) K(\rho, r, t) d \rho+\partial_{t} \int_{t-r}^{t+r} f(\rho) K(\rho, r, t) d \rho, \quad r \neq 0,
$$

where

$$
\begin{aligned}
K(\rho, r, t) & =\frac{(-1)^{m}}{2 m !}\left(\frac{\rho}{r}\right)^{2 m+1}\left(\frac{\partial}{\partial \rho} \frac{1}{2 \rho}\right)^{m} \phi^{m}(\rho, r, t) \\
\phi(\rho, r, t) & =(r-t+\rho)(r+t-\rho)
\end{aligned}
$$

If we set $v(r, t)=r^{2 m+1} u^{0}(r, t)$, then the desired estimates for $u^{0}$ is transformed into the following estimates for $v$ by making use of Leibnitz' rule as $\partial_{r}^{j}\left(r^{j} u_{0}\right)(r, t)=\partial_{r}^{j}\left(r^{-2 m-1+j} v(r, t)\right)$. 
Lemma 2.3. Let $0<t \leq 2 r$ and $r \geq 1$. Suppose (1.8) holds. Then we have

(i) $\quad l<\kappa<l+1, \quad 0 \leq l \leq m, \quad \mu=\kappa-l$ :

$$
\left|\partial_{r}^{j} v(r, t)\right| \leq C \varepsilon\langle t+r\rangle^{1-\mu} r^{m+\max (m-1-j, 0)} \quad \text { for } \quad 0 \leq j \leq m
$$

(ii) $\quad \kappa=l+1, \quad 0 \leq l \leq m$ :

$$
\left|\partial_{r}^{j} v(r, t)\right| \leq C \varepsilon r^{m+\max (m-l-j, 0)} \Psi_{j-(m-l)}(r, t) \quad \text { for } \quad 0 \leq j \leq m
$$

(iii) $\quad m+1<\kappa, \quad \nu=\kappa-(m+1)$ :

$$
\left|\partial_{r}^{j} v(r, t)\right| \leq C \varepsilon r^{m}\langle t-r\rangle^{-\nu} \quad \text { for } \quad 0 \leq j \leq m
$$

Proof: For the sake of simplicity, we assume $f \equiv 0$. From (2.15) we have

$$
\partial_{r}^{j} v(r, t)=\int_{t-r}^{t+r} g(\rho) \partial_{r}^{j} K_{0}(\rho, r, t) d \rho+R_{j}^{+}(r, t)+R_{j}^{-}(r, t)
$$

where we have set $K_{0}(\rho, r, t)=r^{2 m+1} K(\rho, r, t), R_{0}^{ \pm}(r, t)=0$ and for $1 \leq j \leq m$

$$
R_{j}^{ \pm}(r, t)=\sum_{s=0}^{j-1} \partial_{r}^{s}\left[\left.\left(g(\rho) \partial_{r}^{j-1-s} K_{0}(\rho, r, t)\right)\right|_{\rho=t \pm r}\right] .
$$

Since $\partial_{r}^{j} K_{0}(\rho, r, t)$ is odd in $\rho$ (see for instance [13]), we get

$$
\partial_{r}^{j} v(r, t)=\int_{|t-r|}^{t+r} g(\rho) \partial_{r}^{j} K_{0}(\rho, r, t) d \rho+R_{j}^{+}(r, t)+R_{j}^{-}(r, t),
$$

In the following lemma and corollary, we prove preliminary results for $K_{0}(\rho, r, t)$.

Lemma 2.4. Let $0 \leq i, k \leq m$ and $\alpha=0,1$ and set $\gamma=i+\alpha+k$. Then we have

$$
\left.\partial_{t}^{\alpha} \partial_{r}^{i} \partial_{\rho}^{k} \phi(\rho, r, t)\right|_{\rho=t \pm r}= \begin{cases}0 & \text { if } 0 \leq \gamma \leq m-1 \text { or } \gamma \geq 2 m+1 \\ C r^{2 m-\gamma} & \text { if } m \leq \gamma \leq 2 m\end{cases}
$$

Moreover when $|t-r| \leq \rho \leq t+r$, we have

$$
\begin{aligned}
& \left|\partial_{t}^{\alpha} \partial_{r}^{i} \partial_{\rho}^{k} \phi^{m}(\rho, r, t)\right| \leq C r^{2 m-\gamma}, \\
& \left|\partial_{t}^{\alpha} \partial_{r}^{i} \partial_{\rho}^{k} \phi^{m}(\rho, r, t)\right| \leq C r^{m} \rho^{m-\gamma} \quad \text { if } \quad 0 \leq \gamma \leq m .
\end{aligned}
$$

Proof: For $|t-r| \leq \rho \leq t+r$, we have both $0 \leq r-t+\rho \leq 2 r, 2 \rho$ and $0 \leq r+t-\rho \leq 2 r$. Therefore we have (2.20). The others can be shown less hard. 
Corollary 2.5. Let $0 \leq i \leq m$ and $\alpha=0,1$. Suppose that both $|t-r| \leq \rho \leq t+r$ and $\rho \leq 3 r$ hold. Then we have

$$
\left|\partial_{t}^{\alpha} \partial_{r}^{i} K_{0}(\rho, r, t)\right| \leq C r^{m} \rho^{m+1-(i+\alpha)} \quad \text { for } \quad|t-r| \leq \rho \leq t+r
$$

Proof: Note that

hence we have

$$
K_{0}(\rho, r, t)=\sum_{k=0}^{m} C_{k} \rho^{1+k} \partial_{\rho}^{k} \phi^{m}(\rho, r, t)
$$

$$
\partial_{t}^{\alpha} \partial_{r}^{i} K_{0}(\rho, r, t)=\sum_{k=0}^{m} C_{k} \rho^{1+k} \partial_{t}^{\alpha} \partial_{r}^{i} \partial_{\rho}^{k} \phi^{m}(\rho, r, t) .
$$

Set $\gamma=i+\alpha+k$. When $0 \leq \gamma \leq m$, by (2.20) we have

$$
\left|\rho^{1+k} \partial_{t}^{\alpha} \partial_{r}^{i} \partial_{\rho}^{k} \phi^{m}(\rho, r, t)\right| \leq C r^{m} \rho^{1+m-(i+\alpha)} .
$$

When $m+1 \leq \gamma \leq 2 m$, by (2.19) we have

$$
\left|\rho^{1+k} \partial_{t}^{\alpha} \partial_{r}^{i} \partial_{\rho}^{k} \phi^{m}(\rho, r, t)\right| \leq C \rho^{1+k} r^{2 m-\gamma} \leq C \rho^{1+m-(i+\alpha)} r^{m}
$$

because $r^{m-\gamma} \leq(\rho / 3)^{m-\gamma}$. Thus we have proved the corollary.

Applying (2.18) with $i=j-1-s$ and $\alpha=0$ to $R_{j}^{ \pm}$defined in (2.16), we obtain

Corollary 2.6. Let $1 \leq j \leq m$. Suppose that $0<t \leq 2 r$. Then we have

$$
\left|R_{j}^{ \pm}(r, t)\right| \leq C r^{m}|t \pm r|^{m+1-j} \sum_{s=0}^{j-1}\left|(t \pm r)^{1+s} g^{(s)}(t \pm r)\right| \text {. }
$$

End of the Proof of Lemma 2.3: Let $0<t \leq 2 r$ and $r \geq 1$. By (1.8), $\left|\rho^{s} g^{(s)}(\rho)\right| \leq$ $C \varepsilon(\rho)^{-1-\kappa}, 0 \leq s \leq m$. It then follows from (2.17), (2.21) with $i=j, \alpha=0$ and (2.23) that

$$
\begin{aligned}
& |v(r, t)| \leq C \varepsilon r^{m} \int_{|t-r|}^{t+r}\langle\rho\rangle^{m-\kappa} d \rho, \\
& \left|\partial_{r}^{j} v(r, t)\right| \leq C \varepsilon r^{m} \int_{|t-r|}^{t+r}\langle\rho\rangle^{m-j-\kappa} d \rho \\
& \quad+C \varepsilon r^{m}\langle t+r\rangle^{m+1-j-\kappa}+C \varepsilon r^{m}\langle t-r\rangle^{m+1-j-\kappa}, \quad 1 \leq j \leq m .
\end{aligned}
$$

First we deal with $(i)$. Let $0 \leq j \leq m-l$. Since $\langle\rho\rangle^{m-j-\kappa} \leq\langle 3 r\rangle^{m-j-l}\langle\rho\rangle^{-\mu}$ for $|t-r| \leq$ $\rho \leq t+\underline{r} \leq 3 r$, we have by $(2.24)$

$$
\begin{aligned}
\left|\partial_{r}^{j} v(r, t)\right| \leq & C \varepsilon r^{2 m-j-l} \int_{|t-r|}^{t+r}\langle\rho\rangle^{-\mu} d \rho \\
& +C \varepsilon r^{2 m-j-l}\langle t+r\rangle^{1-\mu}+C \varepsilon r^{2 m-j-l}\langle t-r\rangle^{1-\mu}
\end{aligned}
$$


which implies the desired estimate, because $1-\mu>0$. When $m-l+1 \leq j \leq m$, we have $m-j-\kappa \leq-\mu$. Therefore we have by $(2.24)$

$$
\left|\partial_{r}^{j} v(r, t)\right| \leq C \varepsilon r^{m} \int_{|t-r|}^{t+r}\langle\rho\rangle^{-\mu} d \rho+C \varepsilon r^{m}\langle t+r\rangle^{1-\mu}+C \varepsilon r^{m}\langle t-r\rangle^{1-\mu}
$$

hence we obtain $(i)$.

Next we consider (ii). If we take $\delta>0$ so small that $m-l-\delta>0$, we have

$$
\left|\partial_{r}^{j} v(r, t)\right| \leq C \varepsilon r^{2 m-j-1-\delta} \int_{|t-r|}^{t+r}\langle\rho\rangle^{-1+\delta} d \rho+C \varepsilon r^{2 m-j-l} \text { for } 0 \leq j \leq m-l-1 .
$$

When $m-l \leq j \leq m$, we have

$$
\left|\partial_{r}^{j} v(r, t)\right| \leq C \varepsilon r^{m} \int_{|t-r|}^{t+r}\langle\rho\rangle^{-1} d \rho+C \varepsilon r^{m}
$$

hence we obtain (ii). One derive (iii) less hard, so we omit the detail.

End of the Proof of Proposition 2.2: Lemma 2.3 and (2.14) yield (2.12), hence we have shown the proposition.

3. Basic A Priori Estimates. As will be seen later, a solution to the initial value problem (1.11), (1.7) is furnished by a solution of the following integral equation associated with the problem:

$$
u(r, t)=u^{0}(r, t)+L(u)(r, t) \text { in } \mathbb{R} \times[0, \infty),
$$

where $u^{0}$ is given by (2.11) and we have set for $u \in X$

$$
\begin{aligned}
& L(u)(r, t)=\int_{0}^{t} d \tau \int_{-1}^{1} H(t-\tau+r \sigma, \tau) \psi(\sigma) d \sigma \\
& H(\rho, \tau)=\frac{1}{2 m !}\left(\frac{1}{2 \rho} \frac{\partial}{\partial \rho}\right)^{m}\left(\rho^{2 m+1} G(u)(\rho, \tau)\right), \quad \psi(\sigma)=\left(1-\sigma^{2}\right)^{m}
\end{aligned}
$$

$G$ is the function in (1.11) and $X$ is defined above Proposition 2.2.

In this section, we examine basic estimates of the above integral operator for $u \in X$. Since $p>p_{0}(n)$ by $\left(H_{1}\right)$, we have $\kappa_{0}=2 /(p-1)<(m+1) p-1$. Therefore without loss of generality we may assume

$$
\kappa \leq(m+1) p-1
$$

because if (1.8) holds for some $\tilde{\kappa}$, then (1.8) necessarily holds for any $\kappa \leq \tilde{\kappa}$. To begin with, we shall show the following: 
Lemma 3.1. Suppose $\left(H_{1}\right)$ holds. If we set $F(\rho, \tau)=G(u)(\rho, \tau)$ for $u \in X$, then $\rho^{j} \partial_{\rho}^{j} F(\rho, \tau)$ is continuous on $\mathbb{R} \times[0, \infty), 0 \leq j \leq m$. Moreover we have for $\|u\| \leq 1$

(i) $\quad l<\kappa<l+1, \quad 0 \leq l \leq m, \quad \mu=\kappa-l$ :

$$
\begin{gathered}
\sum_{j=0}^{m-l}\left|\rho^{j} \partial_{\rho}^{j} F(\rho, \tau)\right| \leq C\|u\|^{p}\langle\tau+|\rho|\rangle^{-p \mu}\langle\rho\rangle^{-p l} \\
\sum_{j=m-l+1}^{m}\left|\rho^{j} \partial_{\rho}^{j} F(\rho, \tau)\right|\langle\rho\rangle^{-j} \leq C\|u\|^{p}\langle\tau+|\rho|\rangle^{-p \mu}\langle\rho\rangle^{-p l-m+l}
\end{gathered}
$$

(ii) $\quad \kappa=l+1, \quad 0 \leq l \leq m, \quad q=\max (p, m)$ :

$$
\begin{gathered}
\sum_{j=0}^{m-l-1}\left|\rho^{j} \partial_{\rho}^{j} F(\rho, \tau)\right| \leq C\|u\|^{p}\langle\tau+|\rho|\rangle^{-p}\langle\rho\rangle^{-p l} \\
\sum_{j=m-1}^{m}\left|\rho^{j} \partial_{\rho}^{j} F(\rho, \tau)\right|\langle\rho\rangle^{-j} \leq C\|u\|^{p}\langle\tau+|\rho|\rangle^{-p}\langle\rho\rangle^{-p l-m+l}\left(\log \frac{\langle\tau+\mid \rho\rangle}{\langle\tau-|\rho|\rangle}\right)^{q}
\end{gathered}
$$

(iii) $\quad m+1<\kappa, \quad \nu=\kappa-(m+1)$ :

$$
\sum_{j=0}^{m}\left|\rho^{j} \partial_{\rho}^{j} F(\rho, \tau)\right|\langle\rho\rangle^{-j} \leq C|| u||^{p}\langle\tau+|\rho|\rangle^{-p}\langle\rho\rangle^{-p m}\langle\tau-|\rho|\rangle^{-p \nu}
$$

Proof: First we shall show the regularity result for $F(\rho, \tau)$. Let $\rho \neq 0$. By the chain rule, we get for $1 \leq j \leq m$

$$
\partial_{\rho}^{j} F(\rho, \tau)=\sum_{k=1}^{j} G^{(k)}(u)(\rho, \tau) \sum_{\beta \in \mathbb{N}^{k}} C_{\beta} \prod_{i=1}^{k} \partial_{\rho}^{\beta_{i}} u(\rho, \tau),
$$

where $\beta=\left(\beta_{1}, \cdots, \beta_{k}\right)$ with $\sum_{i=1}^{k} \beta_{i}=j, C_{\beta}$ is a constant and $\mathbb{N}$ denote the set of positive integers. Therefore we have

$$
\rho^{j} \partial_{\rho}^{j} F(\rho, \tau)=\sum_{k=1}^{j} G^{(k)}(u)(\rho, \tau) \sum_{\beta \in \mathbb{N}^{k}} C_{\beta} \prod_{i=1}^{k} \rho^{\beta_{i}} \partial_{\rho}^{\beta i} u(\rho, \tau),
$$

hence $\rho^{j} \partial_{\rho}^{j} F(\rho, \tau) \in C(\mathbb{R} \times[0, \infty))$ for $u \in X$.

Moreover since $|u(\rho, \tau)| \leq\|u\| \leq 1$, by $\left(H_{1}\right)$ we obtain

$$
\left|\rho^{j} \partial_{\rho}^{j} F(\rho, \tau)\right| \leq C \sum_{k=1}^{j} A|u(\rho, \tau)|^{(p-k)+} \sum_{\beta \in \mathbb{N}^{k}} \prod_{i=1}^{k}\left|\rho^{\beta i} \partial_{\rho}^{\beta i} u(\rho, \tau)\right|
$$


where we have set $\alpha_{+}=\max (\alpha, 0)$ for $\alpha \in \mathbb{R}$. We shall only show the estimates $(i)$, because the others are proved analogously. When $0 \leq j \leq m-l$, since $\left|\rho^{\beta_{i}} \partial_{\rho}^{\beta_{i}} u(\rho, \tau)\right| \leq$ $C\|u\|\langle\tau+|\rho|\rangle^{-\mu}\langle\rho\rangle^{-1}$ for any $\beta_{i}$, we obtain the desired estimate. Moreover since the estimate for $\rho^{j+1} \partial_{\rho}^{j+1} F(\rho, \tau)$ is not worse than $\rho^{j} \partial_{\rho}^{j} F(\rho, \tau)$, one can derive the desired estimate for $m-l+1 \leq j \leq m$. This completes the proof of the lemma.

Since $H(\rho, \tau)=\sum_{j=0}^{m} C_{j} \rho^{1+j} \partial_{\rho}^{j} F(\rho, \tau)$, we can easily derive the following:

Corollary 3.2. Under the assumption of Lemma 3.1, $H(\rho, \tau) \in C(\mathbb{R} \times[0, \infty))$ for $u \in X$. Moreover we have for $\|u\| \leq 1$

(i) $\quad l<\kappa<l+1, \quad 0 \leq l \leq m, \quad \mu=\kappa-l$ :

$$
|H(\rho, \tau)| \leq C\|u\|^{p}\langle\tau+|\rho|\rangle^{-p \mu}\langle\rho\rangle^{-p l+l+1}
$$

(ii) $\quad \kappa=l+1, \quad 0 \leq l \leq m, \quad q=\max (p, m)$ :

$$
|H(\rho, \tau)| \leq C\|u\|^{p}\langle\tau+|\rho|\rangle^{-p}\langle\rho\rangle^{-p l+l+1}\left(1+\log \frac{\langle\tau+\mid \rho\rangle}{\langle\tau-|\rho|\rangle}\right)^{q}
$$

(iii) $\quad m+1<\kappa, \quad \nu=\kappa-(m+1)$ :

$$
|H(\rho, \tau)| \leq C|| u \|^{p}\langle\tau+|\rho|\rangle^{-p}\langle\rho\rangle^{-p m+m+1}\langle\tau-|\rho|\rangle^{-p \nu}
$$

Our goal of this section is the following:

Proposition 3.3. Suppose $\left(H_{1}\right)$ and (3.2) hold. Let $u$ belong to $X$ and be even in $r$. Then $L(u) \in X$ and $r^{2} L(u)(r, t) \in C^{2}(\mathbb{R} \times[0, \infty))$. Moreover $L(u)$ satisfies

$$
r^{2}\left(\partial_{t}^{2}-\partial_{r}^{2}-\frac{n-1}{r} u_{r}\right) L(u)=r^{2} G(u) \text { in } \mathbb{R} \times[0, \infty)
$$

and zero initial data. Besides $L(u)(r, t)$ is even in $r$. Furthermore if $\|u\| \leq 1$, we have

$$
\|L(u)\| \leq C_{1}\|u\|^{p} \Phi_{2-(p-1) \kappa}(t+|r|),
$$

where $C_{1}$ is a numerical constant depending only on $m, \kappa, p$ and $A$. In addition, we have set $\Phi_{\alpha}(s)=\max \left(1,\langle s\rangle^{\alpha}\right)$ for $s, \alpha \in \mathbb{R}$.

Proof: $\quad$ For $0 \leq \tau \leq t$ we set

$$
E(r, t ; \tau)=\int_{-1}^{1} H(t-\tau+r \sigma, \tau) \psi(\sigma) d \sigma
$$


Since $H(\rho, \tau) \in C(\mathbb{R} \times[0, \infty))$ by Lemma 3.2, applying Lemma 2.1 to $E(r, t ; \tau)$, we have $\partial_{r}^{j}\left(r^{j} E\right)(r, t ; \tau) \in C(\mathbb{R} \times[0, \infty) \times[0, \infty)), 0 \leq j \leq m$ and $\partial_{r, t}^{\alpha}\left(r^{2} E\right)(r, t ; \tau) \in C(\mathbb{R} \times[0, \infty) \times$ $[0, \infty))$ for $|\alpha| \leq 2$. Besides $E(r, t ; \tau)$ is even in $r$. Moreover for any fixed $\tau, E(r, t ; \tau)$ satisfies both (2.4) and at $\tau=t$

$$
E(r, t ; t)=0, \quad \partial_{t} E(r, t ; t)=G(u)(r, t)
$$

Note that (3.5) implies

$$
\begin{aligned}
& \partial_{t} L(r, t)=\int_{0}^{t} \partial_{t} E(r, t ; \tau) d \tau \\
& \partial_{t}^{2} L(r, t)=\int_{0}^{t} \partial_{t}^{2} E(r, t ; \tau) d \tau+G(u)(r, t)
\end{aligned}
$$

Hence all these facts lead to the assertions of the proposition except the estimate (3.3). (See also [13], Proposition 3.4).

In what follows we concentrate on proving the estimate (3.3). At first, we prepare Lemma 3.4 through Corollary 3.8 below which will are refined version of Lemma 4.8 in Kubo and $\mathrm{K}$. Kubota [14]. Here we also note that $p_{0}(n)>(n+1) /(n-1)=(m+2) /(m+1)$.

Lemma 3.4. Suppose (3.2) and $p>(m+2) /(m+1)$ hold. Let $y \geq 0, q=\max (p, m)$ and $\nu=\kappa-(m+1)$. When $\kappa \neq 2 /(p-1)$, we have

$$
\begin{aligned}
\int_{-y}^{y}\langle x+y\rangle^{1-(p-1) \kappa}\left(2+\log \left(\frac{1+y}{1+|x|}\right)\right)^{q} d x & \leq C \Phi_{2-(p-1) \kappa}(y) \\
\int_{-y}^{y}\langle x+y\rangle^{m+2-(m+1) p}\langle x\rangle^{-p \nu} d x & \leq C\langle y\rangle^{-\nu} \Phi_{2-(p-1) \kappa}(y) \text { if } \quad \nu>0
\end{aligned}
$$

Moreover when $\kappa=2 /(p-1)$, we further assume $p>p_{0}(n)$, accordingly $\kappa<(m+1) p-1$. Let $0<\delta_{0}<1$ be so small that $(m+1) p-\kappa-1-\delta_{0}>0$ and $(m+1) p-(m+2)-\delta_{0}>0$. Then we have for $0<\delta<\delta_{0}$

$$
\begin{aligned}
\int_{-y}^{y}\langle x+y\rangle^{-1+\delta}\left(2+\log \left(\frac{1+y}{1+|x|}\right)\right)^{q} d x & \leq C\langle y\rangle^{\delta}, \\
\int_{-y}^{y}\langle x+y\rangle^{m+2-(m+1) p+\delta}\langle x\rangle^{-p \nu} d x \leq C\langle y\rangle^{-\nu+\delta} & \text { if } \quad \nu>0 .
\end{aligned}
$$

where $C$ is independent of $y$.

Proof: First we shall show (3.7a). To begin with, we claim that for $a, b \geq 0$ with $a+b \neq 1$

$$
\int_{-y}^{y}\langle x\rangle^{-a}\langle x+y\rangle^{-b} d x \leq C \Phi_{1-(a+b)}(y)
$$


where $C$ is a constant depending only on $a+b$. Indeed, (3.8) immediately follows from

$$
\langle x\rangle^{-a}\langle x+y\rangle^{-b} \leq\langle x\rangle^{-a-b}+\langle x+y\rangle^{-a-b}
$$

We now divide the $x$-integral into $I_{1}$ and $I_{2}$ :

$$
I_{1}=\int_{-y}^{-y / 2}\langle x\rangle^{-p \nu}\langle x+y\rangle^{m+2-(m+1) p} d x, \quad I_{2}=\int_{-y / 2}^{y}\langle x\rangle^{-p \nu}\langle x+y\rangle^{m+2-(m+1) p} d x
$$

Then we have

$$
I_{1} \leq\left\langle\frac{y}{2}\right\rangle^{-\nu} \int_{-y}^{y}\langle x\rangle^{-(p-1) \nu}\langle x+y\rangle^{m+2-(m+1) p} d x
$$

Since $(p-1) \nu>0,(m+1) p-(m+2)>0$ and $(p-1) \nu+(m+1) p-(m+2)=(p-1) \kappa-1 \neq 1$, by (3.8) we get (3.7a) for $I_{1}$. As to $I_{2}$, we have

$$
I_{2} \leq\left\langle\frac{y}{2}\right\rangle^{-\nu} \int_{-y}^{y}\langle x\rangle^{-p \nu}\langle x+y\rangle^{\kappa+1-(m+1) p} d x
$$

Since $p \nu>0$ and $(m+1) p-(\kappa+1) \geq 0$ by (3.2), we obtain (3.7a) as above.

Next we consider (3.6a). We divide the $x$-integral into $I_{1}$ and $I_{2}$ :

$$
\begin{aligned}
& I_{1}=\int_{-y}^{-y / 2}\left(2+\log \left(\frac{1+y}{1+|x|}\right)\right)^{q}\langle x+y\rangle^{1-(p-1) \kappa} d x \\
& I_{2}=\int_{-y / 2}^{y}\left(2+\log \left(\frac{1+y}{1+|x|}\right)\right)^{q}\langle x+y\rangle^{1-(p-1) \kappa} d x
\end{aligned}
$$

Then we have

$$
I_{1} \leq(2+\log 2)^{q} \int_{-y}^{y}\langle x+y\rangle^{1-(p-1) \kappa} d x
$$

which implies (3.6a). As to $I_{2}$, we have

$$
I_{2} \leq\left\langle\frac{y}{2}\right\rangle^{1-(p-1) \kappa} \int_{-y}^{y}\left(2+\log \left(\frac{1+y}{1+|x|}\right)\right)^{q} d x
$$

We note that $x$-integral is majored by $C y$ as in Tsutaya [24], Proposition 4.3. Indeed, let $k$ be a positive integers such that $q \leq k$ and set

$$
f_{k}(y)=\int_{0}^{y}\left(2+\log \left(\frac{1+y}{1+x}\right)\right)^{k} d x .
$$

Integrating by parts, we then have

$$
\begin{aligned}
f_{k}(y) & =\left[x\left(2+\log \left(\frac{1+y}{1+x}\right)\right)^{k}\right]_{x=0}^{x=y}+k \int_{0}^{y} \frac{x}{1+x}\left(2+\log \left(\frac{1+y}{1+x}\right)\right)^{k-1} d x \\
& \leq 2^{k} y+k f_{k-1}(y)
\end{aligned}
$$

Therefore we obtain $f_{k}(y) \leq C_{k} y$, because $f_{0}(y)=y$. Hence we obtain (3.6a).

Analogously to obtain (3.6a), (3.7a), we get (3.6b), (3.7b) respectively. This completes the proof of the lemma. 
Corollary 3.5. Suppose (3.2) and $p>p_{0}(n)$. Let $0<r \leq 1$ or $t \geq 2 r>0$ and set $\rho_{ \pm}=t-\tau \pm r$. Then we have

(i) $\quad l<\kappa<l+1, \quad 0 \leq l \leq m, \quad \mu=\kappa-l$ :

$$
I_{1}=\int_{0}^{t} d \tau \int_{\rho_{-}}^{\rho_{+}}\langle\tau+|\rho|\rangle^{-p \mu}\langle\rho\rangle^{-p l+l+1} d \rho \leq C r\langle t+r)^{-\mu} \Phi_{2-(p-1) \kappa}(t+r)
$$

(ii) $\quad \kappa=l+1, \quad 0 \leq l \leq m, \quad q=\max (p, m)$ :

$$
I_{2}=\int_{0}^{t} d \tau \int_{\rho_{-}}^{\rho_{+}}\langle\tau+|\rho|\rangle^{-p}\langle\rho\rangle^{-p l+l+1}\left(1+\log \frac{\langle\tau+|\rho|\rangle}{\langle\tau-|\rho|\rangle}\right)^{q} d \rho \leq C r\langle t+r\rangle^{-1} \Phi_{2-(p-1) \kappa}(t+r)
$$

(iii) $\quad m+1<\kappa \leq(m+1) p-1, \quad \nu=\kappa-(m+1)$ :

$I_{3}=\int_{0}^{t} d \tau \int_{\rho_{-}}^{\rho_{+}}\langle\tau+|\rho|\rangle^{-p}\langle\rho\rangle^{-p m+m+1}\langle\tau-|\rho|\rangle^{-p \nu} d \rho \leq C r\langle t+r\rangle^{-1-\nu} \Phi_{2-(p-1) \kappa}(t+r)$.

Proof: First we shall show (i). Setting $\tilde{\rho}_{-}=\max \left(\rho_{-}, 0\right)$, we get

$$
I_{1} \leq 2 \int_{0}^{t} d \tau \int_{\tilde{\rho}_{-}}^{\rho_{+}}\langle\tau+\rho\rangle^{-p \mu}\langle\rho\rangle^{-p l+l+1} d \rho \leq \int_{(t-r)_{+}}^{t+r}\langle\xi\rangle^{-p \mu} d \xi \int_{-\xi}^{\xi}\left\langle\frac{\xi+\eta}{2}\right\rangle^{-p l+l+1} d \eta
$$

where we have changed the variables by $\xi=\rho+\tau, \eta=\rho-\tau$. Note that

$$
-p l+l+1=p \mu-\mu+1-(p-1) \kappa .
$$

Since $p \mu-\mu>0$, the $\eta$-integral is dominated by $C\langle\xi\rangle^{p \mu-\mu} \Phi_{2-(p-1) \kappa}(\xi)$ if $1-(p-1) \kappa \neq-1$. Otherwise, we slightly modify the above procedure as follows: Taking $\delta>0$ so small that $p \mu-\mu-\delta>0$ and employing $-p l+l+1=p \mu-\mu-\delta+1-(p-1) \kappa+\delta$ instead of (3.10), we get the same upper bound of the $\eta$-integral. Therefore we have

$$
I_{1} \leq C \int_{(t-r)_{+}}^{t+r}\langle\xi\rangle^{-\mu} \Phi_{2-(p-1) \kappa}(\xi) d \xi \leq C r\langle t+r\rangle^{-\mu} \Phi_{2-(p-1) \kappa}(t+r)
$$

because $\langle\xi\rangle$ is equivalent to $\langle t+r\rangle$ for $(t-r)_{+} \leq \xi \leq t+r$ if either $0<r \leq 1$ or $t \geq 2 r>0$.

Next we consider $I_{2}$ (resp. $I_{3}$ ). Noting that

$$
-p l+l+1=p-1+1-(p-1) \kappa \quad(\text { resp. }-p m+m+1=p-1+m+2-(m+1) p)
$$

and using (3.6) (resp. (3.7)), we obtain the desired estimate. 
Corollary 3.6. Suppose (3.2) and $p>p_{0}(n)$. Let $0<t \leq 2 r$. Then we have

(i) $\quad l<\kappa<l+1, \quad 0 \leq l \leq m, \quad \mu=\kappa-l$ :

$$
I_{1}^{\prime} \leq C\langle t+r\rangle^{1-\mu} \Phi_{2-(p-1) \kappa}(t+r)
$$

(ii) $\quad \kappa=l+1, \quad 0 \leq l \leq m$ :

$$
I_{2}^{\prime} \leq C\left(1+\log \frac{\langle t+r\rangle}{\langle t-r\rangle}\right) \Phi_{2-(p-1) \kappa}(t+r) ;
$$

(iii) $m+1<\kappa \leq(m+1) p-1, \quad \nu=\kappa-(m+1)$ :

$$
I_{3}^{\prime} \leq C\langle t-r\rangle^{-\nu} \Phi_{2-(p-1) \kappa}(t+r)
$$

where $I_{k}^{\prime}, 1 \leq k \leq 3$ are equal to $I_{k}$ with $\rho_{-}$replaced by $\left|\rho_{-}\right|$.

Proof: Here we shall indicate only the point which is different from the proof of Corollary 3.5. We have instead of (3.9)

$$
I_{1}^{\prime} \leq \int_{|t-r|}^{t+r}\langle\xi\rangle^{-p \mu} d \xi \int_{-(t-r)}^{\xi}\left\langle\frac{\xi+\eta}{2}\right\rangle^{-p l+l+1} d \eta
$$

hence we obtain

$$
I_{1}^{\prime} \leq C \int_{|t-r|}^{t+r}\langle\xi\rangle^{-\mu} \Phi_{2-(p-1) \kappa}(\xi) d \xi \leq C \Phi_{2-(p-1)_{\kappa}}(t+r) \int_{|t-r|}^{t+r}\langle\xi\rangle^{-\mu} d \xi .
$$

We omit the further detail.

The following two corollaries will be used only for the case $r \geq 1$.

Corollary 3.7. Suppose (3.2) and $p>p_{0}(n)$. Let $t \geq 2 r>0$. Then we have

(i) $\quad l<\kappa<l+1, \quad 0 \leq l \leq m, \quad \mu=\kappa-l$ :

$$
J_{1}^{ \pm}=\int_{0}^{t}\left\langle\rho_{ \pm}\right\rangle^{-p l+l+1} d \tau \leq C\langle t \pm r\rangle^{p \mu-\mu} \Phi_{2-(p-1) \kappa}(t \pm r)
$$

(ii) $\quad \kappa=l+1, \quad 0 \leq l \leq m$ :

$$
J_{2}^{ \pm}=\int_{0}^{t}\left\langle\rho_{ \pm}\right\rangle^{-p l+l+1} d \tau \leq C\langle t \pm r\rangle^{p-1} \Phi_{2-(p-1) \kappa}(t \pm r)
$$

(iii) $-m+1<\kappa \leq(m+1) p-1, \quad \nu=\kappa-(m+1):$

$$
J_{3}^{ \pm}=\int_{0}^{t}\left\langle\rho_{ \pm}\right\rangle^{-p m+m+1}\left\langle\tau-\left|\rho_{ \pm}\right|\right\rangle^{-p \nu} d \tau \leq C\langle t \pm r\rangle^{p-1-\nu} \Phi_{2-(p-1) \kappa}(t \pm r) .
$$


Proof: We shall consider only $J_{i}^{-}, 1 \leq i \leq 3$, because the others can be handled less hard. First we deal with $J_{1}^{-}$. Changing the variable by $\rho=t-\tau-r$, we have

$$
J_{1}^{-} \leq \int_{-r}^{t-r}\langle\rho\rangle^{-p l+l+1} d \rho \leq 2 \int_{0}^{t-r}\langle\rho\rangle^{-p l+l+1} d \rho
$$

because $t \geq 2 r$. By (3.10), we get the desired estimate. Similarly one can deal with $J_{2}^{-}$by virtue of (3.11).

Next we consider $J_{3}^{-}$by dividing it as follows:

$$
J_{3}^{-}=\int_{0}^{t-r} d \tau+\int_{t-r}^{t} d \tau \equiv Q_{-}+Q_{+}
$$

Changing the variable by $\rho=t-\tau-r$, we have

$$
\begin{aligned}
& Q_{-}=\int_{0}^{t-r}\langle\rho\rangle^{-p m+m+1}\langle t-r-2 \rho\rangle^{-p \nu} d \rho \\
& Q_{+}=\langle t-r\rangle^{-p \nu} \int_{-r}^{0}\langle\rho\rangle^{-p m+m+1} d \rho .
\end{aligned}
$$

By (3.11) we have

$$
\begin{aligned}
Q_{-} & \leq\langle t-r\rangle^{p-1} \int_{0}^{t-r}\langle\rho\rangle^{m+2-(m+1) p}\langle t-r-2 \rho\rangle^{-p \nu} d \rho \\
& \leq C\langle t-r\rangle^{p-1-\nu} \Phi_{2-(p-1) \kappa}(t-r),
\end{aligned}
$$

where we have used (3.7) as $x=2 p-t+r$. On the other hand, noting that $-p m+m+1=$ $(1+\nu)(p-1)+1-(p-1) \kappa$, we obtain

$$
Q_{+} \leq\langle t-r\rangle^{-p \nu} \int_{0}^{t-r}\langle\rho\rangle^{-p m+m+1} d \rho \leq C\langle t-r\rangle^{-p \nu+(1+\nu)(p-1)} \Phi_{2-(p-1) \kappa}(t-r),
$$

which implies the desired estimate for $J_{3}^{-}$, hence we have shown the corollary.

Corollary 3.8. Suppose (3.2) and $p>p_{0}(n)$. Let $0<t \leq 2 r$. Then we have

(i) $\quad l<\kappa<l+1, \quad 0 \leq l \leq m, \quad \mu=\kappa-l$ :

$$
J_{1}^{ \pm \prime}=\int_{0}^{t}\left\langle\tau+\left|\rho_{ \pm}\right|\right\rangle^{-p \mu}\left\langle\rho_{ \pm}\right\rangle^{-p l+l+2} d \tau \leq C\langle t+r\rangle^{1-\mu} \Phi_{2-(p-1) \kappa}(t+r)
$$

(ii) $\quad \kappa=l+1, \quad 0 \leq l \leq m$ :

$$
J_{2}^{ \pm^{\prime}}=\int_{0}^{t}\left\langle\tau+\left|\rho_{ \pm}\right|\right\rangle^{-p}\left\langle\rho_{+}\right\rangle^{-p l+l+2} d \tau \leq C\left(1+\log \frac{\langle t+r\rangle}{\langle t-r\rangle}\right) \Phi_{2-(p-1) \kappa}(t+r) ;
$$

(iii) $-m+1<\kappa \leq(m+1) p-1, \quad \nu=\kappa-(m+1)$ :

$$
{J_{3}^{ \pm \prime}}^{\prime}=\int_{0}^{t+r}\left\langle\tau+\left|\rho_{ \pm}\right|\right\rangle^{-p}\left\langle\rho_{ \pm}\right\rangle^{-p m+m+1}\left\langle\tau-\left|\rho_{ \pm}\right|\right\rangle^{-p \nu} d \tau \leq C\langle t \pm r\rangle^{-\nu} \Phi_{2-(p-1) \kappa}(t+r) \text {. }
$$


Proof: We shall consider only $J_{i}^{ \pm^{\prime}}, 1 \leq i \leq 3$. It follows

$$
J_{1}^{-\prime}=\int_{0}^{t}\left\langle\rho_{-}\right\rangle^{-p \pi+t+2} d \tau \leq 2 \int_{0}^{r}\langle\rho\rangle^{-p \kappa+l+2} d \rho
$$

where we have changed the variable by $\rho=t-\tau-r$. Since $-p \kappa+l+2=1-\mu+1-(p-1) \kappa$, we get the desired estimate.

Next we consider $J_{2}^{-\prime}$ by dividing it as follows:

$$
J_{2}^{-\prime}=\int_{0}^{(t-r)_{+}} d \tau+\int_{(t-r)_{+}}^{t} d \tau \equiv Q_{-}^{\prime}+Q_{+}^{\prime}
$$

Changing the variable by $\rho=t-\tau-r$, we have

$$
\begin{aligned}
& Q_{-}^{\prime}=\langle t-r\rangle^{-p} \int_{0}^{t-r}\langle\rho\rangle^{-p l+l+2} d \rho \\
& Q_{+}^{\prime}=\int_{-r}^{t-r-(t-r)_{+}}\langle t-r-2 \rho\rangle^{-p}\langle\rho\rangle^{-p l+l+2} d \rho .
\end{aligned}
$$

Since $\langle t-r\rangle^{-p} \leq\langle t-r\rangle^{-\delta}\langle\rho\rangle^{-p+\delta}$ for $0 \leq \rho \leq t-r$, we have

$$
Q_{-}^{\prime} \leq\langle t-r\rangle^{-\delta} \int_{0}^{t-r}\langle\rho\rangle^{1-(p-1) \kappa+\delta} d \rho
$$

where if $2-(p-1) \kappa=0$, we take $\delta>0$ so small that $-p+\delta<0$, otherwise $\delta=0$. This inequality yields $Q_{-}^{\prime} \leq C \Phi_{2-(p-1) \kappa}(t-r)$. On the other hand, since $t-r-(t-r)_{+}=-(r-t)_{+}$, we get

$$
Q_{+}^{\prime} \leq \int_{(r-t)_{+}}^{r}\langle t-r+2 \rho\rangle^{-p}\langle\rho\rangle^{-p l+l+2} d \rho \leq \Phi_{2-(p-1) \kappa}(r) \int_{(r-t)_{+}}^{r}\langle t-r+2 \rho\rangle^{-1} d \rho,
$$

because $-p l+l+2=2-(p-1) \kappa+p-1$ and $t-r+\rho \geq 0$ for $\rho \geq(r-t)_{+}$. Therefore we have $Q_{+}^{\prime} \leq C\left(1+\log \frac{\langle t+r\rangle}{\langle t-r\rangle}\right) \Phi_{2-(p-1) \kappa}(r)$, hence obtain the desired estimate for $J_{2}^{-^{\prime}}$.

Finally we deal with $J_{3}^{-\prime}$.

$$
J_{3}^{-\prime}=\int_{0}^{(t-r)_{+}} d \tau+\int_{(t-r)_{+}}^{t} d r \equiv Q_{-}^{\prime \prime}+Q_{+}^{\prime \prime}
$$

Changing the variable by $\rho=t-\tau-r$, we have $Q_{-}^{\prime \prime}=\langle t-r\rangle^{-p} Q_{-}$, by (3.12). Since (3.13) is still valid for $r \leq t \leq 2 r$, we get $Q_{-}^{\prime \prime} \leq\langle t-r\rangle^{-1-\nu} \Phi_{2-(p-1) \kappa}(t-r)$. As to $Q_{+}^{\prime \prime}$, we have

$$
Q_{+}^{\prime \prime} \leq\langle t-r\rangle^{-p \nu} \int_{(t-r)_{+}}^{t}\left\langle\rho_{-}\right\rangle^{-(m+1)^{p+m+1}} d \tau \leq\langle t-r\rangle^{-p \nu},
$$

because $m+2-(m+1) p<0$. Therefore we have $J_{3}^{-1} \leq C\langle t-r\rangle^{-\nu} \Phi_{2-(p-1) \kappa}(t-r)$.

We are now in a position to estimate $L(r, t)$. 
Lemma 3.9. Suppose $\left(H_{1}\right)$ and (3.2) hold. Let $0<r \leq 1$. Then we have for $u \in X$ with $\|u\| \leq 1$

(i) $\quad l<\kappa<l+1, \quad 0 \leq l \leq m, \quad \mu=\kappa-l$ :

$$
\left|\partial_{r}^{j}\left(r^{j} L\right)(r, t)\right| \leq C\|u\|^{p}\langle t\rangle^{-\mu} \Phi_{2-(p-1) \kappa}(t), \quad 0 \leq j \leq m
$$

(ii) $\quad \kappa=l+1, \quad 0 \leq l \leq m$ :

$$
\left|\partial_{r}^{j}\left(r^{j} L\right)(r, t)\right| \leq C\|u\|^{p}\langle t\rangle^{-1} \Phi_{2-(p-1) \kappa}(t), \quad 0 \leq j \leq m
$$

$$
\begin{aligned}
m+1 & <\kappa \leq(m+1) p-1, \quad \nu=\kappa-(m+1): \\
& \left|\partial_{r}^{j}\left(r^{j} L\right)(r, t)\right| \leq C\|u\|^{p}\langle t\rangle^{-1-\nu} \Phi_{2-(p-1) \kappa}(t), \quad 0 \leq j \leq m .
\end{aligned}
$$

Proof: It follows from (3.4) and (2.3a) that for $0 \leq j \leq m$

$$
\partial_{r}^{j}\left(r^{j} E\right)(r, t ; \tau)=\int_{-1}^{1} H(t-\tau+r \sigma, \tau)(-\sigma)^{j} \psi^{(j)}(\sigma) d \sigma
$$

hence for $r \neq 0$ we have

$$
\left|\partial_{r}^{j}\left(r^{j} L\right)(r, t)\right| \leq \int_{0}^{t}\left|\partial_{r}^{j}\left(r^{j} E\right)(r, t ; \tau)\right| d \tau \leq C r^{-1} \int_{0}^{t} d \tau \int_{\rho_{-}}^{\rho_{+}}|H(\rho, \tau)| d \rho,
$$

where we have changed the variable by $\rho=t-\tau+r \sigma$. Employing Corollaries 3.2 and 3.5, we obtain the desired estimates. This completes the proof of the lemma.

When $r \geq 1$, setting $L_{0}(r, t)=r^{2 m+1} L(r, t)$, the desired estimates for $L(r, t)$ are transformed into Lemmas 3.10 and 3.12 .

Lemma 3.10. Suppose $\left(H_{1}\right)$ and (3.2) hold. Let $t \geq 2 r$. $\geq 2$ and $u \in X$ be even in $r$. If $\|u\| \leq 1$, we have

(i) $\quad l<\kappa<l+1, \quad 0 \leq l \leq m, \quad \mu=\kappa-l$ :

$\left|\partial_{r}^{j} L_{0}(r, t)\right| \leq C\|u\|^{p}\langle t+r\rangle^{-\mu} r^{m+1+\max (m-l-j, 0)} \Phi_{2-(p-1) \kappa}(t+r), \quad 0 \leq j \leq m ;$

(ii) $\quad \kappa=l+1, \quad 0 \leq l \leq m$ :

$$
\left|\partial_{r}^{j} L_{0}(r, t)\right| \leq C\|u\|^{p}\langle t+r\rangle^{-1} r^{m+1+\max (m-1-j, 0)} \Phi_{2-(p-1) \kappa}(t+r), \quad 0 \leq j \leq m ;
$$

(iii) $\quad m+1<\kappa \leq(m+1) p-1, \quad \nu=\kappa-(m+1)$ :

$$
\left|\partial_{r}^{j} L_{0}(r, t)\right| \leq C\|u\|^{p}\langle t+r\rangle^{-1-\nu} r^{m+1} \Phi_{2-(p-1) \kappa}(t+r), \quad 0 \leq j \leq m .
$$


Proof: We split the $\tau$-integral of $\partial_{r}^{j} L_{0}(r, t)$ into

$$
A_{j}(r, t)=\int_{0}^{t-2 r} \partial_{r}^{j}\left(r^{2 m+1} E(r, t ; \tau)\right) d \tau, \quad B_{j}(r, t)=\int_{t-2 r}^{t} \partial_{r}^{j}\left(r^{2 m+1} E(r, t ; \tau)\right) d \tau .
$$

Analogously to obtaining (2.15), we have by (3.4)

$$
r^{2 m+1} E(r, t ; \tau)=\int_{\rho_{-}}^{\rho_{+}} F(\rho, \tau) K_{0}(\rho, r, t-\tau) d \rho,
$$

where $F(\rho, \tau)=G(u)(\rho, \tau)$ and $K_{0}(\rho, r, t)=r^{2 m+1} K(\rho, r, t)$ with $K$ a given function in (2.15). If we set for $0 \leq k \leq m$

$$
w_{k}(r, t, \tau)=\int_{\rho_{-}}^{\rho_{+}} \rho^{1+k} F(\rho, \tau) \partial_{\rho}^{k} \phi^{m}(\rho, r, t-\tau) d \rho,
$$

the result is $0 \leq j \leq m$

$$
A_{j}(r, t)=\sum_{k=0}^{m} C_{k} \int_{0}^{t-2 r} \partial_{\tau}^{j} w_{k}(r, t, \tau) d \tau
$$

by virtue of (2.22). Note that

$$
\rho_{-} \geq r \text { for } \tau \leq t-2 r .
$$

We shall show only $(i)$, because the others can be handled analogously. By Corollary $3.5(i)$, it is enough to show for $0 \leq j \leq m$

$$
\left|\partial_{r}^{j} w_{k}(r, t, \tau)\right| \leq C|| u||^{p} r^{m+\max (m-l-j, 0)} \int_{\rho_{-}}^{\rho_{+}}\langle\tau+|\rho|\rangle^{-p \mu}\langle\rho\rangle^{-p l+l+1} d \rho .
$$

Case 1: $0 \leq j \leq m-l$.

When $0 \leq k \leq l$, we have

$$
\partial_{r}^{j} w_{k}(r, t, \tau)=\int_{\rho_{-}}^{\rho_{+}} \rho^{1+k} F(\rho, \tau) \partial_{r}^{j} \partial_{\rho}^{k} \phi^{m}(\rho, r, t-\tau) d \rho
$$

because $\left.\partial_{r, \rho}^{\alpha} \phi^{m}(\rho, r, t-\tau)\right|_{\rho=\rho_{ \pm}}=0$ for $|\alpha| \leq m-1$. By (2.19) and (3.15), we have

$$
\left|\partial_{r}^{j} \partial_{\rho}^{k} \phi^{m}(\rho, r, t-\tau)\right| \leq C r^{2 m-j-k} \leq C r^{2 m-j-l} \rho^{l-k} \text { for } \rho_{-} \leq \rho \leq \rho_{+}
$$

When $l+1 \leq k \leq m$, by integration by parts in (3.14) we have

$$
\partial_{r}^{j} w_{k}(r, t, \tau)=(-1)^{k-l} \int_{\rho_{-}}^{\rho_{+}} \partial_{\rho}^{k-l}\left(\rho^{1+k} F(\rho, \tau)\right) \partial_{r}^{j} \partial_{\rho}^{l} \phi^{m}(\rho, r, t-\tau) d \rho .
$$

By (2.19) we have

$$
\left|\partial_{r}^{j} \partial_{\rho}^{l} \phi^{m}(\rho, r, t-\tau)\right| \leq C r^{2 m-j-l} .
$$

As to the factor $\rho^{1+k} F(\rho, \tau)$, we have 
Lemma 3.11. Suppose $\left(H_{1}\right)$ holds. Let $l<\kappa<l+1$ with $0 \leq l \leq m$ and $0 \leq i \leq k \leq m$. Then we have for $u \in X$ with $\|u\| \leq 1$

$$
\begin{aligned}
& \left|\partial_{\rho}^{i}\left(\rho^{1+k} F(\rho, \tau)\right)\right| \leq C\|u\|^{p}\langle\tau+|\rho|\rangle^{-p \mu}\langle\rho\rangle^{-p l+k-i+1} \quad \text { if } 0 \leq i \leq m-l \\
& \left|\partial_{\rho}^{i}\left(\rho^{1+k} F(\rho, \tau)\right)\right| \leq C\|u\|^{p}\langle\tau+|\rho|\rangle^{-p \mu}\langle\rho\rangle^{-p l+l+1} \quad \text { if } \quad m-l+1 \leq i \leq k .
\end{aligned}
$$

Proof: (3.13a) follows from Lemma $3.1(i)$ with $0 \leq j \leq m-l$. When $m-l+1 \leq i \leq k$, we have the same estimate (3.13a) with $i=m-l$, hence we get (3.13b).

Substituting (3.18a) and (3.19a) with $i=0$ into (3.17a), we get (3.16) for $0 \leq k \leq l$. By (3.17b), (3.18b) and (3.19a) with $i=k-l$, we obtain (3.16) for $l+1 \leq k \leq m$.

Case 2: $m-l+1 \leq j \leq m$.

First we suppose $j+k \leq m$. From (3.14) we have (3.17a). By (2.19), (3.15) and $r \geq 1$, we have for $\rho_{-} \leq \rho \leq \rho_{+}$

$$
\left|\partial_{r}^{j} \partial_{\rho}^{k} \phi^{m}(\rho, r, t-\tau)\right| \leq C r^{2 m-j-k} \leq C r^{m} \rho^{m-j-k} \leq C r^{m}\langle\rho\rangle^{l-k-1}
$$

because $0 \leq m-j-k \leq l-k-1$. Substituting (3.18c) and (3.19a) with $i=0$ into (3.17a), we get (3.16) in this case.

Next we suppose $j+k \geq m+1$. By integration by parts in (3.14), we have

$$
\partial_{r}^{j} w_{k}(r, t, \tau)=(-1)^{k+j-m} \int_{\rho_{-}}^{\rho_{+}} \partial_{\rho}^{k+j-m}\left(\rho^{1+k} F(\rho, \tau)\right) \partial_{r}^{j} \partial_{\rho}^{m-j} \phi^{m}(\rho, r, t-\tau) d \rho
$$

By (2.19) we have

$$
\left|\partial_{r}^{j} \partial_{\rho}^{m-j} \phi(\rho, r, t-\tau)\right| \leq C r^{m}
$$

Noting that $k+j-m \leq k$ and $-p l+k-(k+j-m)+1 \leq-p l+l$ for $j \geq m-l+1$, we get by $(3.19)$

$$
\left|\partial_{\rho}^{k+j-m}\left(\rho^{1+k} F(\rho, \tau)\right)\right| \leq\left. C\|u\|\right|^{p}\langle\tau+|\rho|\rangle^{-p \mu}\langle\rho\rangle^{-p l+l+1}
$$

By (3.17c), (3.18d) and (3.20), we get (3.16). We thus obtain the desired estimate for $A_{j}$.

Next we consider $B_{j}$. Analogously to obtaining (2.17), we have for $0 \leq j \leq m$

$$
\partial_{r}^{j}\left(r^{2 m+1} E(r, t ; \tau)\right)=W_{j}(r, t, \tau)+R_{j}^{+}(r, t-\tau ; \tau)+R_{j}^{-}(r, t-\tau ; \tau)
$$


where $R_{j}^{ \pm}(r, t-\tau ; \tau)$ is equal to $R_{j}^{ \pm}(r, t)$ given by (2.16) with $g(\rho)$ replaced by $F(\rho, \tau)$ and

$$
W_{j}(r, t, \tau)=\int_{|\rho-|}^{\rho_{+}} F(\rho, \tau) \partial_{\tau}^{j} K_{0}(\rho, r, t-\tau) d \rho,
$$

because $F(\rho, \tau)=G(u)(\rho, \tau)$ is even in $\rho$ and $K_{0}(\rho, r, t-\tau)$ is odd in $\rho$. Setting for $0 \leq j \leq m$

$$
P_{j}^{ \pm}(r, t)=\int_{t-2 r}^{t} R_{j}^{ \pm}(r, t-\tau ; \tau) d \tau
$$

we have

$$
B_{j}(r, t)=\int_{t-2 r}^{t} W_{j}(r, t, \tau) d \tau+P_{j}^{+}(r, t)+P_{j}^{-}(r, t)
$$

Note that $\left|\rho_{ \pm}\right| \leq 3 r$ for $t-2 r \leq \tau \leq t$.

As to $W_{j}$, by Corollary $3.5(i)$ we have only to show for $0 \leq j \leq m$

$$
\left|W_{j}(r, t, \tau)\right| \leq C\|u\|^{p} r^{m+\max (m-1-j, 0)} \int_{\left|\rho_{-}\right|}^{\rho_{+}}\langle\tau+|\rho|\rangle^{-p \mu}\langle\rho\rangle^{-p l+l+1} d \rho .
$$

Let $\left|\rho_{-}\right| \leq \rho \leq \rho_{+}$. Then $\rho \leq 3 r$, hence by (2.21) we have

$$
\left|\partial_{r}^{j} K_{0}(\rho, r, t-\tau)\right| \leq C r^{m}|\rho|^{m+1-j}
$$

When $0 \leq j \leq m-l, \rho^{m+1-j}$ is dominated by $(3 r)^{m-j-l}|\rho|^{1+l}$. When $m-l+1 \leq j \leq m$, we have $|\rho|^{m+1-j} \leq\langle\rho\rangle^{l}$. Therefore we have (3.22) by Lemma $3.1(i)$ with $j=0$

Next we consider $P_{j}^{+}$. By Corollary $3.7(i)$, it is enough to show for $1 \leq j \leq m$

$$
\left|P_{j}^{ \pm}(r, t)\right| \leq C\|u\|^{p} r^{m+1+\max (m-j-l, 0)}\langle t \pm r\rangle^{-p \mu} \int_{0}^{t}\left\langle\rho_{ \pm}\right\rangle^{-p l+l+1} d \tau .
$$

Since $\left|\rho_{ \pm}\right| \leq 3 r$, by (2.23) we have for $1 \leq j \leq m$

$$
\left|R_{j}^{ \pm}(r, t-\tau ; \tau)\right| \leq C r^{m}\left|\rho_{ \pm}\right|^{m+1-j} \sum_{s=0}^{j-1}\left|\rho_{ \pm}^{1+s} \partial_{\rho}^{s} F\left(\rho_{ \pm}, \tau\right)\right| .
$$

By Lemma $3.1(i)$ we have

$$
\left|\rho_{ \pm}^{i} \partial_{\rho}^{i-1} F\left(\rho_{ \pm}, \tau\right)\right| \leq\left\{\begin{array}{l}
C\|u\|^{p}\langle t \pm r\rangle^{-p \mu}\left\langle\rho_{ \pm}\right\rangle^{-p l+1} \text { if } 1 \leq i \leq m-l+1 \\
C\|u\|^{p}\langle t \pm r\rangle^{-p \mu}\left\langle\rho_{ \pm}\right\rangle^{-p l-m+l+i} \text { if } m-l+2 \leq i \leq m
\end{array}\right.
$$

because $\tau+\left|\rho_{ \pm}\right| \geq t \pm r$ for $\tau \geq 0$. Substituting (3.26) into (3.25), we have

$$
\left|R_{j}^{ \pm}(r, t-\tau ; \tau)\right| \leq\left\{\begin{array}{l}
C\|u\|^{p} r^{2 m+1-j-l}\langle t \pm r\rangle^{-p \mu}\left\langle\rho_{ \pm}\right\rangle^{-p l+l+1} \quad \text { if } \quad 1 \leq i \leq m-l \\
C\|u\|^{p} r^{m+1}\langle t \pm r\rangle^{-p \mu}\left\langle\rho_{ \pm}\right\rangle^{-p l+l} \text { if } m-l+1 \leq i \leq m
\end{array}\right.
$$

which implies (3.24). This completes the proof of Lemma 3.10.

Finally we consider the case $0<t \leq 2 r$ and $r \geq 1$. 
Lemma 3.12. Suppose $\left(H_{1}\right)$ and (3.2) hold. Let $0<t \leq 2 r, r \geq 1$ and $u \in X$ be even in $r$. If $\|u\| \leq 1$, we have

(i) $\quad l<\kappa<l+1, \quad 0 \leq l \leq m, \quad \mu=\kappa-l$ :

$$
\left|\partial_{r}^{j} L_{0}(r, t)\right| \leq C\|u\|^{p}\langle t+r\rangle^{1-\mu} r^{m+\max (m-1-j, 0)} \Phi_{2-(p-1) \kappa}(t+r), \quad 0 \leq j \leq m
$$

(ii) $\quad \kappa=l+1, \quad 0 \leq l \leq m$ :

$$
\left|\partial_{r}^{j} L_{0}(r, t)\right| \leq C\|u\|^{p} r^{m+\max (m-l-j, 0)} \Psi_{j-(m-l)}(r, t) \Phi_{2-(p-1) \kappa}(t+r), \quad 0 \leq j \leq m
$$

(iii) $\quad m+1<\kappa \leq(m+1) p-1, \quad \nu=\kappa-(m+1)$ :

$$
\left|\partial_{r}^{j} L_{0}(r, t)\right| \leq C\|u\|^{p}(t-r)^{-\nu} r^{m} \Phi_{2-(p-1) \kappa}(t+r), \quad 0 \leq j \leq m
$$

Proof: Since the proof is similar to the treatment for $B_{j}$ defined in below Lemma 3.10 , we shall indicate only the different point from the case. Instead of (3.22), we have

$$
\partial_{r}^{j} L_{0}(r, t)=\int_{0}^{t} W_{j}(r, t, \tau) d \tau+{P_{j}^{+}}^{\prime}(r, t)+P_{j}^{-'}(r, t),
$$

where $W_{j}$ is defined by (3.21) and we have set $P_{j}^{ \pm \prime}(r, t)=\int_{0}^{t} R_{j}^{ \pm}(r, t-\tau ; \tau) d \tau$. Note that (3.23) is still valid in this case, because $\rho \leq 3 r$ for $\left|\rho_{-}\right| \leq \rho \leq \rho_{+}$and $t \leq 2 r$. Moreover similarly to obtaining (3.27), we have

$$
\left|R_{j}^{ \pm}(r, t-\tau ; \tau)\right| \leq\left\{\begin{array}{l}
C\|u\|^{p} r^{2 m-j-l}\left\langle\tau+\left|\rho_{ \pm}\right|\right\rangle^{-p \mu}\left\langle\rho_{ \pm}\right\rangle^{-p l+l+2} \quad \text { if } \quad 1 \leq i \leq m-l \\
C\|u\|^{p} r^{m}\left\langle\tau+\left|\rho_{ \pm}\right|\right\rangle^{-p \mu}\left\langle\rho_{ \pm}\right\rangle^{-p l+l+1} \quad \text { if } \quad m-l+1 \leq i \leq m .
\end{array}\right.
$$

Employing Corollaries 3.6 and 3.8 instead of Corollaries 3.5 and 3.7, we get the desired estimate. This completes the proof of the lemma.

\section{Proof of Theorem 1.2.}

Let $u(r, t) \in X$ be a solution to the integral equation (3.1). If $u$ is even in $r$, it then turn out to be a solution to the initial value problem (1.11), (1.7) by Propositions 2.2 and 3.3. Moreover the uniqueness for such a solution that $u(r, t) \in C(\mathbb{R} \times[0, \infty))$ and $r^{2} u(r, t) \in C(\mathbb{R} \times[0, \infty))$ is derived from a standard argument. (See e.g. John [11], Theorem 4 or Agemi [1], p.156). Therefore we have only to construct a solution to the integral equation (3.1) which belongs to $X$ and is even in $r$. 
We shall introduce an auxiliary norm $\|u\| \|$ for $u \in X$ by

(i) $\quad l<\kappa<l+1, \quad 0 \leq l \leq m, \quad \mu=\kappa-l$ :

$$
\|\| u\|\|=\sum_{j=0}^{m-1} \sup _{(r, t)}\left|r \partial_{r}^{j}\left(r^{j} u\right)(r, t)\right|\langle t+|r|\rangle^{\mu}\langle r\rangle^{\min (l, m-j)-1}
$$

(ii) $\quad \kappa=l+1, \quad 0 \leq l \leq m$ :

$$
\|\| u \|\left|=\sum_{j=0}^{m-1} \sup _{(r, t)}\right| r \partial_{r}^{j}\left(r^{j} u\right)(r, t) \mid\langle t+|r|\rangle\langle r\rangle^{\min (l, m-j)-1} \Psi_{j-(m-l)}^{-1}(r, t) ;
$$

(iii) $\quad \kappa>m+1, \quad \nu=\kappa-(m+1)$ :

$$
\|\| u \|\left|=\sum_{j=0}^{m-1} \sup _{(r, t)}\right| r \partial_{r}^{j}\left(r^{j} u\right)(r, t) \mid\langle t+|r|\rangle\langle r\rangle^{m-j-1}\langle t-|r|\rangle^{\nu} .
$$

To apply the iteration method as in [10], we need the Lipschitz type estimates as follows: Proposition 4.1. Suppose (H) and (3.2) hold. Let $u, v$ be even functions belonging to $X$ with $\|u\|,\|v\| \leq 1$. Then we have

$$
\begin{aligned}
\|L(u)-L(v)\| \mid \leq & C_{2}\|u-v\| \|\left(\|u\|^{p-1}+\|v\|^{p-1}\right) \Phi_{2-(p-1) \kappa}(t+r) \\
\|L(u)-L(v)\| \leq & C_{3}\|u-v\|\left(\|u\|^{p-1}+\left.\|v\|\right|^{p-1}\right) \Phi_{2-(p-1) \kappa}(t+r) \\
& +\left.C_{4}\|u-v\|\right|^{\delta}\left(\|u\|^{m}+\|v\|^{m}\right) \Phi_{2-(p-1) \kappa}(t+r)
\end{aligned}
$$

where $C_{4}=0$ if $p>m+1, \tilde{\delta}=p-m$ if $p>m$ and $\tilde{\delta}=\delta$ if $p \leq m$ with $\delta$ is the number in $\left(H_{2}\right)$ and $\Phi$ is given below (3.3).

Proof: First we consider (4.1). Set $\tilde{F}(\rho, \tau)=G(u)(\rho, \tau)-G(v)(\rho, \tau)$. Since

$$
\tilde{F}(\rho, \tau)=(u-v) \int_{0}^{1} G^{(1)}(u \theta+v(1-\theta)) d \theta
$$

we get the following similarly to Lemma 3.1.

Lemma 4.2. Suppose $\left(H_{1}\right)$ holds. Let $u, v$ belongs to $X$ with $\|u\|,\|v\| \leq 1$. Then

(i) $\quad l<\kappa<l+1, \quad 0 \leq l \leq m, \quad \mu=\kappa-l$ :

$$
\begin{gathered}
\sum_{j=0}^{m-1}\left|\rho^{j+1} \partial_{\rho}^{j} \tilde{F}(\rho, \tau)\right| \leq C M\langle\tau+|\rho|\rangle^{-p \mu}\langle\rho\rangle^{-p l+1} \\
\sum_{j=m-l+1}^{m-1}\left|\rho^{j+1} \partial_{\rho}^{j} \tilde{F}(\rho, \tau)\right|\langle\rho\rangle^{-j} \leq C M\langle\tau+|\rho|\rangle^{-p \mu}\langle\rho\rangle^{-p l-m+l+1}
\end{gathered}
$$


(ii) $\quad \kappa=l+1, \quad 0 \leq l \leq m, \quad q=\max (p, m)$ :

$$
\begin{gathered}
\sum_{j=0}^{m-l-1}\left|\rho^{j+1} \partial_{\rho}^{j} \tilde{F}(\rho, \tau)\right| \leq C M\langle\tau+|\rho|\rangle^{-p}\langle\rho\rangle^{-p l+1}, \\
\sum_{j=m-l}^{m-1}\left|\rho^{j+1} \partial_{\rho}^{j} \tilde{F}(\rho, \tau)\right|\langle\rho\rangle^{-j} \leq C M\langle\tau+|\rho|\rangle^{-p}\langle\rho\rangle^{-p l-m+l+1}\left(\log \frac{\langle\tau+\mid \rho\rangle}{\langle\tau-|\rho|\rangle}\right)^{q} ;
\end{gathered}
$$

(iii) $\quad m+1<\kappa, \quad \nu=\kappa-(m+1)$ :

$$
\sum_{j=0}^{m-1}\left|\rho^{j+1} \partial_{\rho}^{j} \tilde{F}(\rho, \tau)\right|\langle\rho\rangle^{-j} \leq C M\langle\tau+|\rho|\rangle^{-p}\langle\rho\rangle^{-p m+1}\langle\tau-|\rho|\rangle^{-p \nu}
$$

where we have set $M=\|\| u-v\|\|\left(\|u\|^{p-1}+\|v\|^{p-1}\right)$.

For the case $r \geq 1$, proceeding as in the proof of Lemmas 3.10 and 3.12, we obtain the desired estimates. From now on, we consider the case $0<r<1$. Setting $\tilde{H}(\rho, \tau)=$ $\frac{1}{2 m !}\left(\frac{1}{2 \rho} \frac{\partial}{\partial \rho}\right)^{m}\left(\rho^{2 m+1} \tilde{F}(\rho, \tau)\right)$, we have from (3.1)

$$
\tilde{L}(r, t) \equiv L(u)(r, t)-L(v)(r, t)=\int_{0}^{t} d \tau \int_{-1}^{1} \tilde{H}(t-\tau+r \sigma, \tau) \psi(\sigma) d \sigma,
$$

By (2.3a) we have for $0 \leq j \leq m-1$

$$
\partial_{r}^{j}\left(r^{j} \tilde{L}\right)(r, t)=\int_{0}^{t} d \tau \int_{-1}^{1} \tilde{H}(t-\tau+r \sigma, \tau) \psi_{j}(\sigma) d \sigma
$$

where we have set $\psi_{j}(\sigma)=(-\sigma)^{j} \psi^{(j)}(\sigma)$. Changing the variable by $\rho=t-\tau+r \sigma$, we have

$$
\begin{aligned}
r \partial_{r}^{j}\left(r^{j} \tilde{L}\right)(r, t) & =\int_{0}^{t} d \tau \int_{\rho_{-}}^{\rho_{+}} \tilde{H}(\rho, \tau) \psi_{j}(\sigma) d \rho \\
& =-\int_{0}^{t} d \tau \int_{\rho_{-}}^{\rho_{+}} \frac{1}{2 m !}\left(\frac{1}{2 \rho} \frac{\partial}{\partial \rho}\right)^{m-1}\left(\rho^{2 m+1} \tilde{F}(\rho, \tau)\right)\left(\frac{\partial}{\partial \rho} \frac{1}{2 \rho}\right) \psi_{j}(\sigma) d \rho
\end{aligned}
$$

where $\sigma=(\rho-(t-\tau)) / r$ and $\rho_{ \pm}=t-\tau \pm r$. It is easy to see that

$$
\left|\left(\frac{\partial}{\partial \rho} \frac{1}{2 \rho}\right) \psi_{j}(\sigma)\right| \leq C\left(\rho^{-2}+r^{-1} \rho^{-1}\right) \text { for } \rho_{-} \leq \rho \leq \rho_{+} .
$$

Since $\frac{1}{2 m !}\left(\frac{1}{2 \rho} \frac{\partial}{\partial \rho}\right)^{m-1}\left(\rho^{2 m+1} \tilde{F}(\rho, \tau)\right)=\sum_{k=0}^{m-1} C_{k} \rho^{3+k} \partial_{\rho}^{k} \tilde{F}(\rho, \tau)$, we have for $0 \leq j \leq m-1$

$$
\left|r \partial_{r}^{j}\left(r^{j} \tilde{L}\right)(r, t)\right| \leq C \sum_{k=0}^{m-1} C_{k} \int_{0}^{t}\left(I_{1}+I_{2}\right) d \tau
$$

where we have set

$$
\left.\left.I_{1}=\int_{\rho_{-}}^{\rho_{+}} \mid \rho^{1+k} \partial_{\rho}^{k} \tilde{F}(\rho, \tau)\right)\left|d \rho, \quad I_{2}=r^{-1} \int_{\rho_{-}}^{\rho_{+}}\right| \rho^{2+k} \partial_{\rho}^{k} \tilde{F}(\rho, \tau)\right) \mid d \rho .
$$

Employing Lemma 4.2 and Corollary 3.5, we have the desired estimate.

Using the below and proceeding as in section 3, we get (4.2). 
Lemma 4.3. Suppose $(H)$ holds. Let $u, v$ belong to $X$ with $\|u\|,\|v\| \leq 1$. Then Lemma 3.1 with $0 \leq j \leq m-1$ is still valid for $\tilde{F}$. Moreover we have

(i) $\quad l<\kappa<l+1, \quad 0 \leq l \leq m, \quad \mu=\kappa-l$ :

$$
\left|\rho^{m} \partial_{\rho}^{m} \tilde{F}(\rho, \tau)\right| \leq C N\langle\tau+|\rho|\rangle^{-p \mu}\langle\rho\rangle^{-p l+l}+D\langle\tau+|\rho|\rangle^{-p \mu}\langle\rho\rangle^{-p l+l+1} \rho^{-1}
$$

(ii) $\quad \kappa=l+1, \quad 0 \leq l \leq m, \quad q=\max (p, m)$ :

$$
\begin{aligned}
\left|\rho^{m} \partial_{\rho}^{m} \tilde{F}(\rho, \tau)\right| \leq & C N\left\langle\tau+\left.|\rho|\right|^{-p \mu}\langle\rho\rangle^{-p l+l}\left(\log \frac{\langle\tau+\mid \rho\rangle}{\langle\tau-|\rho|\rangle}\right)^{q}\right. \\
& +D\langle\tau+|\rho|\rangle^{-p \mu}\langle\rho\rangle^{-p l+l+1} \rho^{-1}\left(\log \frac{\langle\tau+\mid \rho\rangle}{\langle\tau-|\rho|\rangle}\right)^{q}
\end{aligned}
$$

(iii) $\quad m+1<\kappa, \quad \nu=\kappa-(m+1)$ :

$$
\begin{aligned}
\left|\rho^{m} \partial_{\rho}^{m} \tilde{F}(\rho, \tau)\right| \leq & C N\langle\tau+|\rho|\rangle^{-p \mu}\langle\rho\rangle^{-p l+l}\langle\tau-|\rho|\rangle^{-p \nu} \\
& +D\langle\tau+|\rho|\rangle^{-p \mu}\langle\rho\rangle^{-p l+l+1} \rho^{-1}\langle\tau-|\rho|\rangle^{-p \nu}
\end{aligned}
$$

where we have set $N=\|u-v\|\left(\|u\|^{p-1}+\|v\|^{p-1}\right)$ and $D=0$ if $p>m+1$ and $D=$ \|\|$u-v\|\|^{\bar{\delta}}\left(\|u\|^{m}+\|v\|^{m}\right)$ if $p \leq m+1$ with $\tilde{\delta}$ a number in Proposition 4.1.

Proof: The first assertion is dirived from the argument similar to the proof of Lemma 3.1 by virtue of (4.3). Note that

$$
\begin{aligned}
\rho^{m} \partial_{\rho}^{m} \tilde{F}(\rho, \tau)= & \sum_{k=1}^{m-1}\left\{G^{(k)}(u)(\rho, \tau) \sum_{\beta \in \mathbb{N}^{k}} C_{\beta} \prod_{i=1}^{k} \rho^{\beta_{i}} \partial_{\rho}^{\beta i} u(\rho, \tau)\right. \\
& \left.-G^{(k)}(v)(\rho, \tau) \sum_{\beta \in N^{k}} C_{\beta} \prod_{i=1}^{k} \rho^{\beta_{i}} \partial_{\rho}^{\beta i} v(\rho, \tau)\right\} \\
+ & \left\{G^{(m)}(u)(\rho, \tau)\left(\rho \partial_{\rho} u(\rho, \tau)\right)^{m}-G^{(m)}(v)(\rho, \tau)\left(\rho \partial_{\rho} v(\rho, \tau)\right)^{m}\right\}
\end{aligned}
$$

Using $\left(H_{2}\right)$ and the definition of $\||\cdot|\|$ for the second term, we get the desired estimate.

Proposition 4.4. Suppose (H), (3.2) and $0<\kappa<\kappa_{0}$ hold. Let $u, v$ be as in Proposition 4.1. Then we have (4.1) and (4.2) with $\Phi_{2-(p-1) \kappa}(t+r)$ replaced by $\langle t\rangle^{2-(p-1) \kappa}$.

Proof: When $t \geq r / 2>0$ or $0<r \leq 1$, the conclusion directly follows from Proposition 4.1. So we assume $0<t \leq r / 2$ and $r \geq 1$, from now on. Similarly to (3.28), we get

$$
\partial_{r}^{j}\left(L_{0}(u)(r, t)-L_{0}(v)(r, t)\right)=\int_{0}^{t} \tilde{W}_{j}(r, t, \tau) d \tau+\tilde{P}_{j}^{+^{\prime}}(r, t)+\tilde{P}_{j}^{-{ }^{\prime}}(r, t), \quad 0 \leq j \leq m,
$$


where we have set $L_{0}(u)(r, t)=r^{2 m+1} L(u)(r, t)$ and

$$
\begin{aligned}
\tilde{W}_{j}(r, t, \tau) & =\int_{|\rho-|}^{\rho_{+}} \tilde{F}(\rho, \tau) \partial_{r}^{j} K_{0}(\rho, r, t-\tau) d \rho \\
\tilde{P}_{j}^{ \pm^{\prime}} & =\int_{0}^{t} \sum_{s=0}^{j-1} \partial_{r}^{s}\left[\left.\left(\tilde{F}(\rho, \tau) \partial_{r}^{j-1-s} K_{0}(\rho, r, t-\tau)\right)\right|_{\rho=\rho_{ \pm}}\right] d \tau
\end{aligned}
$$

with $K_{0}$ a function defined above (2.16). Since $K_{0}(\rho, r, t-\tau)$ is odd in $\rho$, we have

$$
\tilde{P}_{j}^{+^{\prime}}+\tilde{P}_{j}^{-^{\prime}}(r, t)=\int_{0}^{t} 2(t-\tau) d \tau \int_{0}^{1} \sum_{s=0}^{j-1} \partial_{r}^{s} Q(r, t, \tau ; \lambda) d \lambda
$$

where we have set

$$
Q(r, t, \tau ; \lambda)=\left.\partial_{\rho}\left[\tilde{F}(\rho, \tau) \partial_{r}^{j-1-s} K_{0}(\rho, r, t-\tau)\right]\right|_{\rho=\bar{\rho}} \text { with } \bar{\rho}=\lambda \rho_{+}+(1-\lambda)\left(-\rho_{-}\right)
$$

As to $\tilde{W}_{j}$, using (2.21) and Lemmas 4.2 and 4.3 with $j=0$ and applying the following, we obtain the desired estimates.

Lemma 4.5. Suppose $p>p_{0}(n)$ holds. Let $0<t \leq r / 2$. Then we have

(i) $\quad l<\kappa<l+1, \quad 0 \leq l \leq m, \quad \mu=\kappa-l$ :

$$
\int_{0}^{t} d \tau \int_{\mid \rho-1}^{\rho_{+}}\langle\tau+\rho\rangle^{-p \mu}\langle\rho\rangle^{-p l+l+1} d \rho \leq C\langle t+r\rangle^{1-\mu}\langle t\rangle^{2-(p-1) \kappa}
$$

(ii) $\quad \kappa=l+1, \quad 0 \leq l \leq m$ :

$$
\int_{0}^{t} d \tau \int_{\mid \rho-1}^{\rho+}\langle\tau+\rho\rangle^{-p}\langle\rho\rangle^{-p l+l+1} d \rho \leq C\langle t\rangle^{2-(p-1) \kappa}
$$

(iii) $\quad m+1<\kappa, \quad \nu=\kappa-(m+1)$ :

$$
\int_{0}^{t} d \tau \int_{\left|\rho_{-}\right|}^{\rho_{+}}\langle\tau+\rho\rangle^{-p}\langle\rho\rangle^{-p m+m+1}\langle\tau-\rho\rangle^{-p \nu} d \rho \leq C\langle t-r\rangle^{-\nu}\langle t\rangle^{2-(p-1) \kappa}
$$

Proof: We shall show only (i). Changing the variables by $\xi=\rho+\tau, \eta=\rho-\tau$, we get

$$
\begin{aligned}
(\rho, \tau)-\text { integral } & \leq \frac{1}{2} \int_{r-t}^{t+r}\langle\xi\rangle^{-p \mu} d \xi \int_{r-t}^{\xi}\left\langle\frac{\xi+\eta}{2}\right\rangle^{-p l+l+1} d \eta \\
& \leq \frac{1}{2} \int_{r-t}^{t+r}\langle\xi\rangle^{-p \mu+1}\left\langle\frac{\xi+r-t}{2}\right\rangle^{-p l+l}(\xi-(r-t)) d \xi \\
& \leq C t^{2}\langle t+r\rangle^{-p \kappa+l+1} \leq C t^{2}\langle t\rangle^{-(p-1) \kappa}\langle t+r\rangle^{1-\mu}
\end{aligned}
$$

because $\langle r-t\rangle$ is equivalent to $\langle t+r\rangle$ for $0<t \leq r / 2$. Hence we get $(i)$.

As to $\tilde{P}_{j}^{+^{\prime}}+\tilde{P}_{j}^{-{ }^{\prime}}$, employing Lemmas $4.2,4.3$ and 4.6 and applying Lemmma 4.7 below, we obtain the desired estimates. 
Lemma 4.6. Let $0<t \leq r$ and $0 \leq \tau \leq t$. For $0 \leq \beta \leq s \leq j-1$ and $\alpha=0,1$, we have

$$
\left|\partial_{r}^{\beta}\left[\left.\partial_{\rho}^{\alpha} \partial_{r}^{j-1-s} K_{0}(\rho, r, t-\tau)\right|_{\rho=\bar{\rho}}\right]\right| \leq C r^{m}(\bar{\rho})^{m+1-j+s-\beta+1-\alpha},
$$

where $\bar{\rho}$ is defined in (4.4) with $0 \leq \lambda \leq 1$.

Proof: We consider only the case $\alpha=0$. By (2.22) we have

$$
\partial_{r}^{\beta}\left[\left.\partial_{r}^{j-1-s} K_{0}(\rho, r, t-\tau)\right|_{\rho=\bar{\rho}}\right]=\sum_{k=0}^{m} C_{k} \sum_{\nu=0}^{\beta} C_{\nu} J_{\nu}
$$

where we have set

$$
J_{\nu}=\partial_{r}^{\beta-\nu}\left(\bar{\rho}^{k+1}\right) \partial_{r}^{\nu}\left(\left.\partial_{r}^{j-1-s} \partial_{\rho}^{k} \phi^{m}(\rho, r, t-\tau)\right|_{\rho=\bar{\rho}}\right), \quad \phi^{m}(\rho, r, t-\tau)=\left(\rho-\rho_{-}\right)^{m}\left(\rho_{+}-\rho\right)^{m} .
$$

Since $\partial_{r}\left(\bar{\rho}-\rho_{-}\right)=2$ and $\partial_{r}\left(\rho_{+}-\bar{\rho}\right)=0$, we have

$$
J_{\nu}=C(\bar{\rho})^{k+1-(\beta-\nu)} \sum_{q=0}^{\gamma} C_{q} \partial_{r}^{\nu}\left(\left(\bar{\rho}-\rho_{-}\right)^{m-(\gamma-q)}\right)\left(\rho_{+}-\bar{\rho}\right)^{m-q},
$$

where we have set $\gamma=j-1-s+k$ and $C_{q}=0$ if $m<q$ or $m<\gamma-q$. Note that $0 \leq \bar{\rho}-\rho_{-} \leq 2 \bar{\rho}$ and $0 \leq \rho_{+}-\bar{\rho} \leq 2 r$, because $0 \leq-\rho_{-} \leq \bar{\rho} \leq \rho_{+}$for $r \geq t$ and $0 \leq \tau \leq t$. Therefore we have

$$
\left|J_{\nu}\right| \leq C \sum_{q=0}^{\gamma} C_{q}(\bar{\rho})^{k+1-(\beta-\nu)+m-(\gamma-q)-\nu} r^{m-q} \leq C \sum_{q=0}^{\gamma}(\bar{\rho})^{m+2-j+s-\beta+q} r^{m-q}
$$

which yields (4.5), because $0 \leq \bar{\rho} \leq 2 r$ in the case.

Lemma 4.7. Suppose $p>p_{0}(n)$ holds. Let $0<t \leq r / 2$ and $\bar{\rho}$ is given in (4.4) with $0 \leq \lambda \leq 1$. Then we have

(i) $\quad l<\kappa<l+1, \quad 0 \leq l \leq m, \quad \mu=\kappa-l$ :

$$
\int_{0}^{t}(t-\tau)\langle\tau+\bar{\rho}\rangle^{-p \mu}\langle\bar{\rho}\rangle^{-p l+l+1} d \tau \leq C(t+r\rangle^{1-\mu}\langle t\rangle^{2-(p-1) \kappa}
$$

(ii) $\quad \kappa=l+1, \quad 0 \leq l \leq m, \quad q=\max (p, m)$ :

$$
\int_{0}^{t}(t-\tau)\langle\tau+\bar{\rho}\rangle^{-p}\langle\bar{\rho}\rangle^{-p l+l+1}\left(1+\log \frac{\langle\tau+\bar{\rho}\rangle}{\langle\tau-\bar{\rho}\rangle}\right)^{q} d \tau \leq C\langle t\rangle^{2-(p-1) \kappa}
$$

(iii) $-m+1<\kappa, \quad \nu=\kappa-(m+1)$ :

$$
\int_{0}^{t}(t-\tau)\langle\tau+\bar{\rho}\rangle^{-p}\langle\bar{\rho}\rangle^{-p m+m+1}\langle\tau-\bar{\rho}\rangle^{-p \nu} d \tau \leq C\langle t-r\rangle^{-\nu}\langle t\rangle^{2-(p-1) \kappa} \text {. }
$$


Proof: Since $\langle\bar{\rho}\rangle,\langle\tau+\bar{\rho}\rangle$ and $\langle\tau-\bar{\rho}\rangle$ are equivalent to $\langle t+r\rangle$ in the case, we get the lemma analogously to obtaining Lemma 4.5 .

End of the Proof of Theorem 1.2: First we prove $T_{\varepsilon}=\infty$ if $\kappa \geq \kappa_{0}$. We define a sequence of functions $\left\{u_{k}\right\}_{k=0}^{\infty}$ by

$$
u_{k+1}=u_{0}+L\left(u_{k}\right) \text { for } k \geq 0, \quad u_{0}=u^{0}
$$

where $u^{0}$ is given in (2.11). By Proposition 2.2, we have $\left\|u_{0}\right\| \leq C_{0} \varepsilon$. Since $\kappa \geq \kappa_{0}$, we have (3.3), (4.1) and (4.2) with $\Phi_{2-(p-1) \kappa}(t+r)$ replaced by 1 . Let $\varepsilon_{2}$ be so small that

$$
2 C_{0} \varepsilon_{2} \leq 1 \text { and } 2^{p+2} C_{5}\left(C_{0} \varepsilon_{2}\right)^{p-1} \leq 1
$$

with $C_{5}=\sum_{i=1}^{4} C_{i}$. Then we find a solution $u \in X$ to the integral equation (3.1) for $0<\varepsilon \leq \varepsilon_{2}$ by following [10], p.257-p.259. (See also [13], Section 5).

Next we treat the case $0<\kappa<\kappa_{0}$. We take a positive number $\varepsilon_{1}$ satisfying

$$
\varepsilon_{1} \leq \varepsilon_{2} \quad \text { and } \quad 2^{p+2} C_{5}(\sqrt{2})^{2-(p-1) \kappa}\left(C_{0} \varepsilon_{1}\right)^{p-1}<1
$$

Since $2-(p-1) \kappa>0$ by $0<\kappa<\kappa_{0}$, there is a number $t_{\varepsilon}>1$ uniqely such that

$$
2^{p+2} C_{5}(\sqrt{2} t)^{2-(p-1) \kappa}\left(C_{0} \varepsilon\right)^{p-1}=1, \quad \text { for } \quad 0<\varepsilon \leq \varepsilon_{1}
$$

Then considering (4.6), for $0<\varepsilon \leq \varepsilon_{1}$ and $0<t \leq t_{\varepsilon}$ we have

$$
2 C_{0} \varepsilon \leq 1 \text { and } 2^{p+2} C_{5}\langle t\rangle^{2-(p-1) \kappa}\left(C_{0} \varepsilon\right)^{p-1} \leq 1
$$

because $\langle t\rangle \leq \sqrt{2} \max (t, 1)$. Hence for $0<\varepsilon \leq \varepsilon_{1}$ we get a local solution by iteration and obtain (1.10) by (4.7), because $T_{\varepsilon} \geq t_{\varepsilon}$.

\section{Acknowledgment}

The author would like to acknowledge Professor Kôji Kubota for his valuable advices and constant encouragement.

\section{REFERENCES}

1. R. Agemi, Blow-up of solutions to nonlinear wave equations in two space dimensions, Manuscripta Math. 73 (1991), 153-162. 
2. R. Agemi and H. Takamura, The lifespan of classical solutions to nonlinear wave equations in two space dimensions, Hokkaido Math. J. 21 (1992), 517-542.

3. F. Asakura, Existence of a global solution to a semi-linear wave equation with slowly decreasing initial data in three space dimensions, Comm. in PDE 11(13) (1986), 1459-1487.

4. Y. Choquet-Bruhat, Global existence for solutions of $\square u=A|u|^{p}$ small initial data, J. Diff. Eq 82 (1989), 98-108.

5. D. Christodoulou, Global solutions of nonlinear hyperbolic equations for small initial data, Comm. Pure Appl. Math. 39 (1986), 267-282.

6. R. Courant and D. Hilbert, Methods of mathematical physics II, Intersience, New York (1963).

7. R. T. Glassey, Blow-up theorems for nonlinear wave equations, Math. Z. 132 (1973), 183-203.

8. R. T. Glassey, Finite-time blow-up for solutions of nonlinear wave equations, Math. Z. 177 (1981), 323-340.

9. R. T. Glassey, Existence in the large for $\square u=F(u)$ in two space dimensions, Math. Z. 178 (1981), 233-261.

10. F. John, Blow-up of solutions of nonlinear wave equations in three space dimensions, Manuscripta Math. 28 (1979), 235-268.

11. F. John, Blow-up for quasi-linear wave equations in three space dimensions, Comm. Pure Appl. Math. 34 (1981), 29-51.

12. T. Kato, Blow-up of solutions of some nonlinear hyperbolic equations, Comm. Pure Appl. 33 (1980), 501-505.

13. H. Kubo, Asymptotic behaviors of solutions to semilinear wave equations with initial data of slow decay, to appear in Math. Method in Appl. Sciences.

14. H. Kubo and K. Kubota, Asymptotic behaviors of radial solutions to semilinear wave equations in odd space dimensions, Hokkaido Univ. Preprint Ser. Math. \#225 (1994).

15. K. Kubota, Existence of a global solution to a semi-linear wave equation with initial data of non-compact support in low space dimensions, Hokkaido Math. J. 22 (1993), 123-180.

16. $\mathrm{Li}$ Ta-tsien and Chen Yun-mei, Initial value problems for nonlinear wave equations, Comm. in P.D.E. 13 (1988), 383-422.

17. $\mathrm{Li}$ Ta-tsien and Yun-mei, Life-span of classical solutions to fully nonlinear wave equations, Comm. in P.D.E. 26 (1991), 909-940.

18. J. Schaeffer, The equation $u_{t t}-\Delta u=|u|^{p}$ for the critical value of $p$, Proc. Roy. Soc. Edinburgh 101 A (1985), 31-44.

19. T. C. Sideris, Nonexistence of global solutions to semilinear wave equations in high dimensions, J. Diff. Eq. 52 (1984), 378-406.

20. H. Takamura, Blow-up for nonlinear wave equations with slowly decaying data, to appear in Math. Z.

21. H. Takamura, Blow-up for semilinear wave equations with slowly decaying data in high dimensions, to appear in J. Diff. and Int. Eq.

22. K. Tsutaya, A global existence theorem for semilinear wave equations with data of non compact support in two space dimensions, Comm. in PDE 17(11 \& 12) (1992), 1925-1954.

23. K. Tsutaya, Global existence theorem for semilinear wave equations with noncompact data in two space dimensions, J. Diff. Eq. 104 (1993), 332-360.

24. K. Tsutaya, Global existence and the life span of solutions of semilinear wave equations with data of non compact support in three space dimensions, to appear in Funkcialaj Ekvacioj.

25. Y. Zhou, Cauchy problem for semilinear wave equations in four space dimensions with small initial data, IDMF PREPRINT 9217. 